\title{
Study on the Extraction Simulation of the Cylindrical Features
}

\author{
Zexiang Zhao $\mathbb{D}^{1},{ }^{1}$ Dongxu Ren, ${ }^{1}$ Xinyu Zhao, ${ }^{2}$ Bin Li, ${ }^{1}$ and Ruyi Liu ${ }^{1}$ \\ ${ }^{1}$ School of Mechatronics Engineering, Zhongyuan University of Technology, Zhengzhou 450007, China \\ ${ }^{2}$ School of Computer Science, Zhongyuan University of Technology, Zhengzhou 450007, China \\ Correspondence should be addressed to Zexiang Zhao; zexiang_zhao@zut.edu.cn
}

Received 22 April 2020; Accepted 15 June 2020; Published 27 July 2020

Academic Editor: Xiaoliang Jin

Copyright (C) 2020 Zexiang Zhao et al. This is an open access article distributed under the Creative Commons Attribution License, which permits unrestricted use, distribution, and reproduction in any medium, provided the original work is properly cited.

\begin{abstract}
The extraction simulation method and theory of cylindrical components were promoted in this paper. According to the manufacturing principle of the cylindrical components, the main influence factors of their precision manufacturing were analyzed, and their extraction models of cylindrical features were built under the analysis of the errors of the slideway and the spindle as well as the parallelism error between the slideway and the spindle rotary axis. The determination principle of modeling parameters of cylindrical features was studied. According to the extraction strategies of the roundness profiles, generatrix profiles, birdcage profile, and single spiral profile, some cylindrical features were simulated by using the established models, and their corresponding global sizes, calculated sizes, and form errors were evaluated and compared. The simulation experiments showed that the simulation models can meet the needs for the research of the relationship among the size tolerance, the form tolerance, the waviness, and the roughness, and can be taken as the theoretic base for the error budgets of the slideway and the rotation spindle in the design of precision machine tools.
\end{abstract}

\section{Introduction}

With the development of precision and ultraprecision technology, a lot of cylindrical components with high precision grade are needed. High-precision turning and grinding technologies have being developed for hard and brittle materials, such as glass, ceramics, and crystal, nonferrous materials, such as copper alloy and aluminum alloy, and ferrous metals, as well as porous materials. These highprecision cylindrical components are usually indicated by their sizes and tolerances [1], form tolerances [2], such as roundness, straightness, and cylindricity, and surface roughness [3]. Their sizes, form errors, and surface roughness are measured by the length measuring instruments based on the two-point method, the form error measuring instruments, and the roughness measuring instruments, respectively. For a fit of hole and shaft with the same nominal size, their respective comprehensive sizes formed by two-point size and form errors rather than only their respective two-point size will have actual influence on their fit characteristic. Therefore, besides two-point size, the global sizes, such as least-squares size, minimum circumscribed size, maximum inscribed size, and minimax size, and the calculated sizes, such as circumference diameter, area diameter, and volume diameter, for a cylindrical component are stipulated in ISO 14405-1 [4], which can be measured by the cylindrical coordinate measuring machine [5]. Global sizes and calculated sizes are the comprehensive sizes of the actual sizes and form errors of the manufactured cylindrical component. For obtaining the global sizes and calculated sizes of a cylindrical component, its profiles should be first extracted by using the measuring machine above, and then the evaluation of the sizes can be done by using the program developed based on the definitions of global sizes and calculated sizes. The extraction strategies and their sampling parameters of the cylindrical profiles will have influence on the measuring results of the global sizes and calculated sizes. If the sampling number of a cylindrical component is less, the extracted profiles may not characterize the actual size of the components, so that the global sizes and calculated sizes cannot be correctly obtained, and if the sampling number of a cylindrical component is too much, some sampling points may be redundant, so that the measuring and evaluation of the sizes may be long and some 
cross errors may be introduced in the long measuring process. Therefore, the extraction strategies and their sampling parameters are studied based on the simulation profiles, which are mainly composed of the simulated slideway's linear error profiles and spindle axis's rotary radial error profiles of the precision machine tool.

\section{Modeling of Surface Profiles of Cylindrical Component}

2.1. Influence Factors of Precision Manufacturing of Cylindrical Component. In the field of precision manufacturing, in order to obtain high-precision surface quality, hard and brittle materials, such as glass, ceramics, and crystal, and nonferrous materials, such as copper alloy and aluminum alloy, are usually turned by single-point diamond turning machines and ferrous metal materials and porous materials are usually ground by the precision grinding machines. In the manufacturing of high-precision cylindrical components, whether or not the high-precision turning or grinding way is used, the influence factors of precision manufacturing of cylindrical components mainly consist of the cutting tool (grinding wheel or turning tool), slideway's straightness error, rotary accuracy of the spindle, parallelism error between the slideway and the spindle's rotary axis, external and internal vibration, temperature, etc. Besides, tool abrasion and cutting force also have much influence on the surface quality. The surface roughness is mainly affected by tool's condition and process parameters, and the surface waviness is generated by vibration and the related factors. For convenience, the influences of slideway straightness, rotary accuracy of the spindle, their parallelism error, and some random errors on the cylindrical component's surface quality are mainly considered in this paper. The schematic of position relationship of the spindle and the slideway is shown in Figure 1.

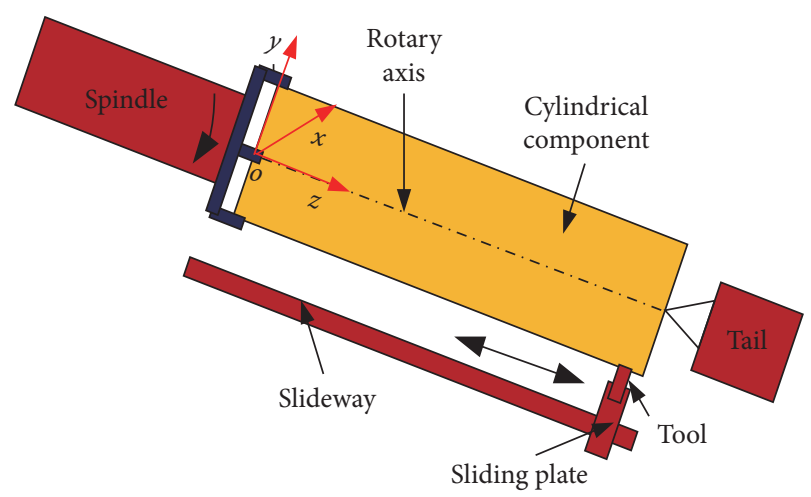

FIGURE 1: Schematic of position relationship of the spindle and the slideway.

2.2. The Error Analysis of the Slideway. In the precision machine tools, the linear slideway, aerostatic bearing slideway, and hydrostatic slideway are usually used. No matter what kind of slideway is adopted in a precision machine tool, there may be some form errors. The slideway's profile in the $z$ direction may be seen in Figure 2-4, which consists of the form error profile, waviness profile, and roughness profile and can be expressed as follows:

$$
\rho_{g p}(z)=\rho_{g f}(z)+\rho_{g w}(z)+\rho_{g r}(z),
$$

where $\rho_{g f}$ is the form error profile, which can be expressed as follows:

$$
\rho_{g f}(z)=W(z)+\sum_{j=1}^{N_{g f}} g_{f w j}(z) \sin \left(\frac{2 \pi z}{j \lambda_{f}}+\theta_{j}\right), \quad z=0 \sim L,
$$

where $W(z)$ can be calculated by the following equation:

$$
W(z)=\left\{\begin{array}{l} 
\pm\left\{\left[R_{A}^{2} \mp(0.5 L-z)^{2}\right]^{1 / 2}-R_{A}+G\right\}, \quad \text { for convex or concave arc profile, } \\
\pm G \operatorname{sine} \frac{\pi z}{L}, \quad \text { for convex or concave sine profile, } \\
\pm 0.5 G \operatorname{sine} \frac{k_{g} \pi z}{L}, \quad k_{g}=2,3,4,5, \text { for sine profile, }
\end{array}\right.
$$

where $G$ is the bow altitude of the arc profile or the magnitude of the half sine wave profile. If $G$ is equal to zero, the slideway form error profile characterized by the first item in equation (2) is an ideal straight line. For convex and concave arc profiles, signs "+" and "-" on the left of the brace are selected, respectively, and signs "-_" and "+" on the left of the parentheses are selected where $z \leq L / 2$ and $z>L / 2$, respectively, as shown in Figure 2, where $L$ is the slideway length and $R_{A}$ is the radius of the arc profile. For the convex half sine profile characterized by the first item in equation (2), sign "+" on the left of $G$ is selected, and for the concave half sine profile, sign "-" on the left of $G$ is selected, as shown in Figure 3. For the sine profile, signs "+" and "-" can be selected according to the actual needs. The second item in equation (2) is also a kind of the form error profile, whose wavelength is $j$ times of the cutoff wavelength $\lambda_{f}$ of the form error profile, $\theta_{j}$ is the initial angle of the $j$ th wave, and $N_{g f}$ is the maximum times of the upper cutoff wavelength $\lambda_{f}$ of the 


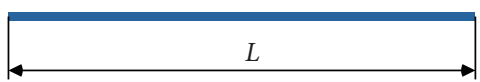

(a)

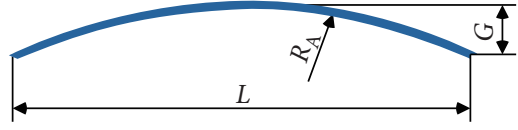

(b)

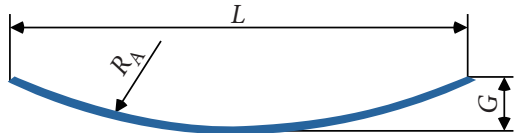

(c)

Figure 2: Schematic of the arc profile of the slideway characterized by the first item in equation (2). (a) Straight. (b) Convex. (c) Concave.

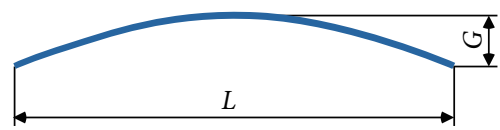

(a)

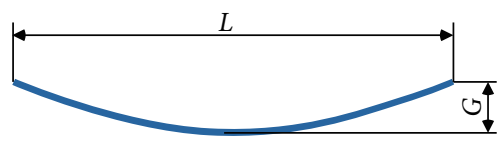

(b)

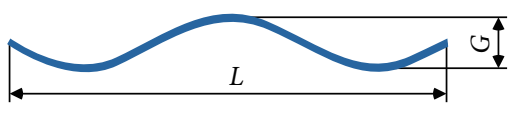

(c)

FIgURE 3: Schematic of the sine profile of the slideway characterized by the first item in equation (2). (a) Convex ( $\left.k_{g}=1\right)$. (b) Concave $\left(k_{g}=1\right)$. (c) Sine profile $\left(k_{g}=2,3, \ldots\right)$.

form error profile, which may be supposed to be five, and the product of times of $N_{g f}$ and $\lambda_{f}$ is equal to or less than the slideway length $L$, as shown in Figure 4.

In equation (1), $\rho_{g w}$ is the waviness profile with a cutoff wavelength $\lambda_{c}$ along the axis of the cylindrical component, which is expressed as follows:

$$
\rho_{g w}(z)=\sum_{j=1}^{N_{g w}} g_{w w j}(z) \sin \left(\frac{2 \pi z}{j \lambda_{c}}+\varphi_{j}\right), \quad z=0 \sim L,
$$

where $g_{w w j}(z)$ is the magnitude of the $j$ th wave in the waviness profile, whose wavelength is $j$ times of the cutoff wavelength $\lambda_{c}$ of the waviness profile, $\varphi_{j}$ is the initial angle of the $j$ th wave, $N_{g w}$ is the maximum times of the cutoff wavelength $\lambda_{c}$, and the product of times of $N_{g w}$ and $\lambda_{c}$ is equal to or less than $\lambda_{f}$.

In equation (1), $\rho_{g r}$ is the roughness profile with a cutoff wavelength $\lambda_{s}$ along the slideway in the $z$ direction, which is expressed as follows:

$$
\rho_{g r}(z)=\sum_{j=1}^{N_{g r}} g_{r w j}(z) \sin \left(\frac{2 \pi z}{j \lambda_{s}}+\vartheta_{j}\right)+\delta(z), \quad z=0 \sim L,
$$

where $g_{r w j}(z)$ is the magnitude of the $j$ th wave in the roughness profile, whose wavelength is $j$ times of the cutoff wavelength $\lambda_{s}$ of the roughness profile, $\vartheta_{j}$ is the initial angle of the $j$ th wave, $N_{g r}$ is the maximum times of the cutoff wavelength $\lambda_{s}$ of the roughness profile, the product of times of $N_{g r}$ and $\lambda_{s}$ is equal to or less than $\lambda_{c}$, and $\delta(z)$ are the random numbers between 0 and $g_{r w 1} / 5$.

The waviness and roughness profiles characterized by equations (4) and (5) are similar to the form profile shown in Figure 4, but their cutoff wavelengths and magnitudes are less than those of the form profiles characterized by equation (2).

2.3. The Rotary Error Analysis of the Spindle. Through the analysis of the roundness profile's compositions introduced by the spindle rotary error, tool wear, vibration, and so on, the roundness profile can be expressed as follows:

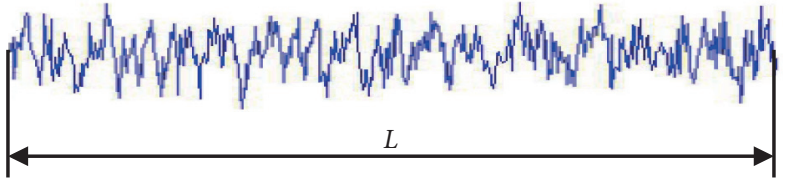

FIgURE 4: The form error profile of the slideway characterized by the second item in equation (2).

$$
\begin{aligned}
\rho_{r p}(z, \psi(z))= & \rho_{r e}(z, \psi(z))+\rho_{r f}(z, \psi(z))+\rho_{r w}(z, \psi(z)) \\
& +\rho_{r r}(z, \psi(z)), \quad z=0 \sim L, \psi(z)=0 \sim 2 \pi,
\end{aligned}
$$

where $\rho_{r e}(z, \psi)$ is the radial size of the cylinder with some eccentricity and multi-sine waves to the rotary spindle axis, shown in Figure 5, which can be expressed as follows:

$$
\begin{aligned}
\rho_{r e}(z, \psi(z))= & \sqrt{\rho_{s k}^{2}(\psi(z))-e^{2}(z) \sin ^{2}(\psi(z)-\phi(z))} \\
& +e(z) \cos (\psi(z)-\phi(z))
\end{aligned}
$$

where $e(z)$ is the eccentricity of the roundness profile, $\phi(z)$ is the eccentricity angle of the roundness profile, $\psi(z)$ is the angle between one point in the roundness profile and the $x$ axis in the xoy coordinate system, and $\rho_{\text {sk }}(\psi(z))$ is the radial size between the rotary axis of the spindle and the circle with $k_{r}$ UPR at the angle $\psi(z)$, where UPR means undulations per round. $\rho_{s k}(\psi(z))$ can be determined as follows:

$$
\begin{array}{r}
\rho_{s k}(\psi(z))=R+A \sin \left(\frac{2 \pi(\psi(z)-m s a(m-1))}{m s a}\right), \\
m=1,2, \ldots, k_{r}, \psi(z)=0 \sim 2 \pi,
\end{array}
$$

where $R$ is the radius of an ideal cylinder, $k_{r}$ is the UPR number in a roundness profile of the cylinder, $A$ is the magnitude of the sine wave profile, and $m s a$ is equal to $2 \pi / k_{r}$. The parameters above can be seen in Figure 5 .

Based on equation (8), the circle profiles with $k_{r}$ UPR can be simulated, as shown in Figure 6.

In equation (6), $\rho_{r f}(z, \psi)$ is the form error profile in the roundness profile, which can be expressed as follows: 


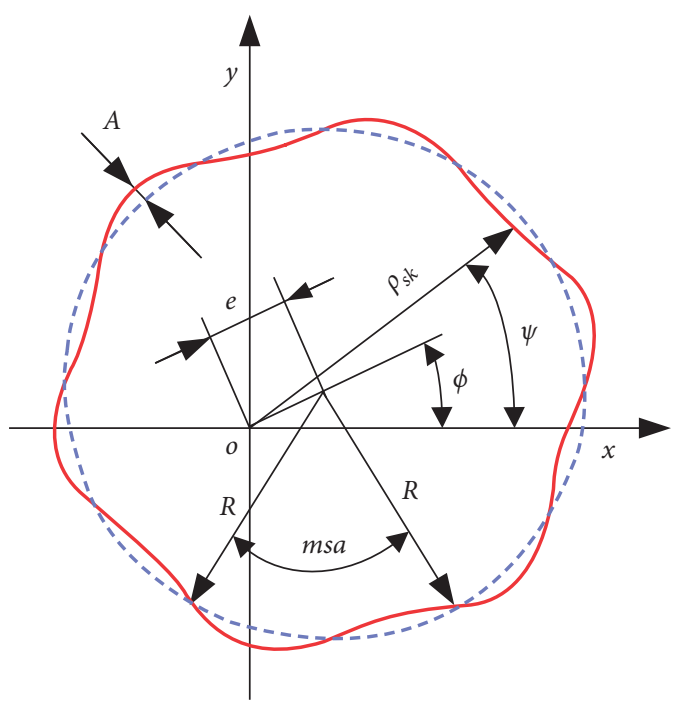

Figure 5: Schematic of the parameters in equations (7) and (8).

$$
\begin{array}{r}
\rho_{r f}(z, \psi(z))=\sum_{j=1}^{N_{r f}} r_{f w j}(z) \sin \left(\frac{\pi D \psi(z)}{j \lambda_{f}}+\zeta_{j}(z)\right), \\
z=0 \sim L, \psi(z)=0 \sim 2 \pi,
\end{array}
$$

where $r_{f w j}(z)$ is the magnitude of the $j$ th wave of the form error profile in the roundness profile, whose wavelength is $j$ times of the cutoff wavelength $\lambda_{f}$ of the form error profile, $\zeta_{j}(z)$ is the initial angle of the $j$ th wave in the roundness profile, $N_{r f}$ is the maximum times of the upper cutoff wavelength $\lambda_{f}$ of the waviness profile, which may be supposed to be five, and the product of times of $N_{r f}$ and $\lambda_{f}$ is equal to or less than the perimeter of the roundness profile.

In equation (6), $\rho_{r w}(z, \psi)$ is the waviness profile in the roundness profile, which can be expressed as follows:

$$
\begin{array}{r}
\rho_{r w}(z, \psi(z))=\sum_{j=1}^{N_{r w}} r_{w w j}(z) \sin \left(\frac{\pi D \psi(z)}{j \lambda_{c}}+\xi_{j}(z)\right), \\
z=0 \sim L, \psi(z)=0 \sim 2 \pi,
\end{array}
$$

where $r_{w w j}(z)$ is the magnitude of the $j$ th wave of the waviness profile in the roundness profile, whose wavelength is $j$ times of the cutoff wavelength $\lambda_{c}$ of the waviness profile, $\xi_{j}(z)$ is the initial angle of the $j$ th wave in the roundness profile, $N_{r w}$ is the maximum times of the upper cutoff wavelength $\lambda_{c}$ of the waviness profile, and the product of times of $N_{r w}$ and $\lambda_{c}$ is equal to or less than $\lambda_{f}$.

In equation (6), $\rho_{r r}(z, \psi)$ is the roughness profile in the roundness profile, which can be expressed as follows:

$$
\begin{array}{r}
\rho_{r r}(z, \psi(z))=\sum_{j=1}^{N_{r r}} r_{r w j}(z) \sin \left(\frac{\pi D \psi(z)}{j \lambda_{s}}+\eta_{j}(z)\right)+\delta(\psi(z)), \\
z=0 \sim L, \psi(z)=0 \sim 2 \pi,
\end{array}
$$

where $r_{r w j}(z)$ is the magnitude of the $j$ th wave of the roughness profile in the roundness profile, whose wavelength is $j$ times of the cutoff wavelength $\lambda_{s}$ of the roughness profile, $\eta_{j}(z)$ is the initial angle of the $j$ th wave, $N_{r r}$ is the maximum times of the cutoff wavelength $\lambda_{s}$ of the roughness profile, the product of times of $N_{r r}$ and $\lambda_{s}$ is equal to or less than $\lambda_{c}$, and $\delta(\psi(z))$ are the random numbers between 0 and $r_{r w 1}(z) / 5$.

In equations (9)-11, $D$ is the diameter of an ideal cylinder.

2.4. The Parallelism Error Analysis of the Slideway to the Spindle Rotary Axis. Owing to that there is the parallelism error of the slideway to the spindle's rotary axis, the fabricated cylinder may be a cone, and the generatrix profile of which may be expressed as follows:

$$
\rho_{s}(z)=S \times \frac{z}{L}+C D, \quad z=0 \sim L,
$$

where $S$ is the slope of the fabricated generatrix, which represents whether the slideway is parallel to the spindle axis, and $C D$ is the intercept of the generatrix in the $z o \rho_{s}$ coordinate system. The parameters in equation (12) are shown in Figure 7.

2.5. The Profile of the Cylinder Gained by Simulation. According to Sections 2.2-2.4, the profile of the cylinder gained by manufacturing simulation can be expressed as follows:

$$
\begin{array}{r}
\rho(z, \psi(z))=\rho_{g p}(z)+\rho_{r p}(z, \psi(z))+\rho_{s}(z), \\
z=0 \sim L, \psi(z)=0 \sim 2 \pi .
\end{array}
$$

Besides the influence factors mentioned above, the modeling of the cylindrical feature's profile simulation does not consider the other influence factors, such as heat deflection, tool wear, and vibration.

\section{Determination of Modeling Parameters of Cylindrical Feature}

For simulation of precision manufacturing, the equations in Section 2 should be discretized. If we use a series of roundness profiles with an equal interval or a single spiral profile to characterize a cylinder's size feature gained by using precision manufacturing simulation, as shown in Figure 8, there are two characteristic parameters, that is, the axial interval $\Delta z_{r}$ of two adjacent roundness profiles and the angular interval $\Delta \psi_{r}$ of two adjacent sampling points in a roundness profile for the size feature in Figure 8(a). The axial interval $\Delta z_{g}$ of two adjacent points in one generatrix profile and the angular interval $\Delta \psi_{g}$ of two adjacent generatrix profiles are two parameters for the generatrix extraction strategy in Figure 8(b). The birdcage extraction strategy in Figure 8(c) needs four parameters, shown in Figures 8(a) and 8 (b), and there are also two characteristic parameters, that is, the pitch $\Delta z_{s}$ of a single spiral or the axial interval $\Delta z_{1}$ of two 


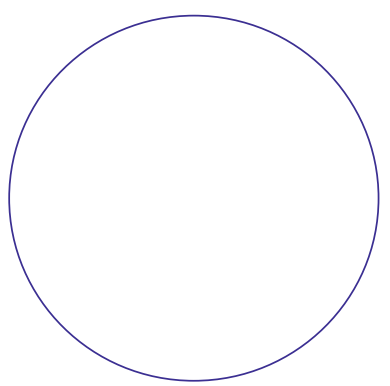

(a)

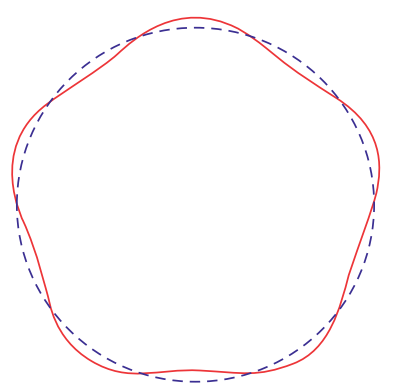

(e)

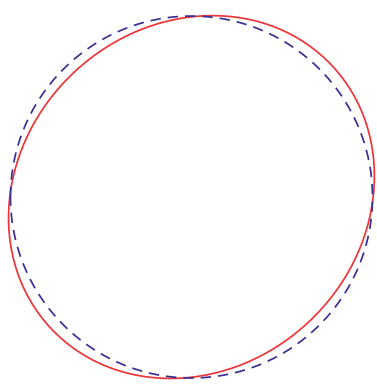

(b)

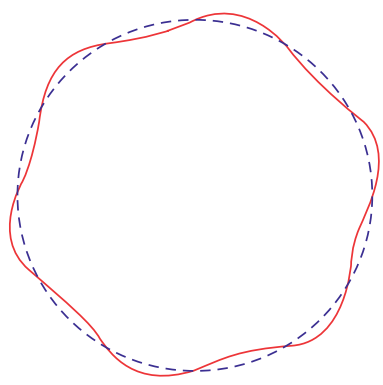

(f)

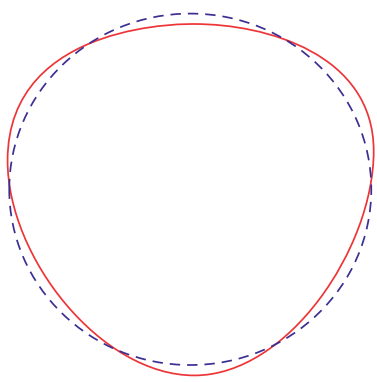

(c)

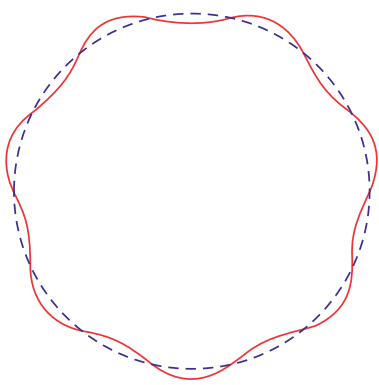

(g)

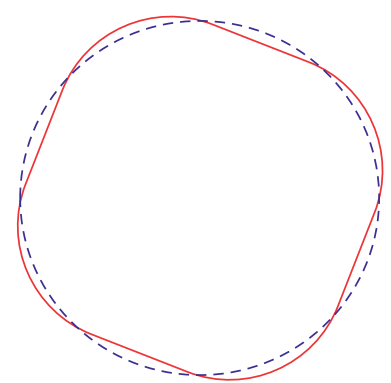

(d)

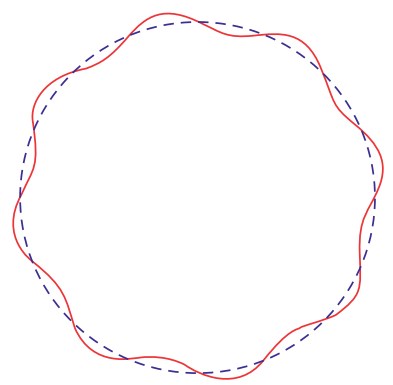

(h)

FIGURE 6: The circle profiles with different $k_{r}$ UPR. (a) $k_{r}=1$. (b) $k_{r}=2$. (c) $k_{r}=3$. (d) $k_{r}=4$. (e) $k_{r}=5$. (f) $k_{\mathrm{r}}=6$. (g) $k_{r}=7$. (h) $k_{r}=8$.

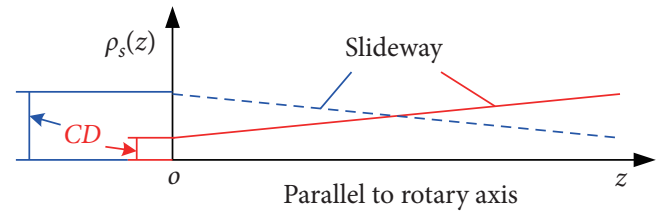

Figure 7: Schematic of the parameters in equation (12).

adjacent sampling points or the angular interval $\Delta \psi_{s}$ of two adjacent sampling points in Figure 8(d). For the need of determining the characteristic parameters, the cylinder's size feature, the cutoff wavelengths of the profiles determined by equation (13) should be analyzed, which are related to the parameters $\Delta z_{r}, \Delta \psi_{r}, \Delta z_{q}, \Delta \psi_{g}, \Delta z_{s}\left(\right.$ or $\left.\Delta z_{1}\right)$, and $\Delta \psi_{s}$. Suppose that the cutoff wavelengths of the form profile, waviness profile, and roughness profile are $\lambda_{f} \lambda_{c}$, and $\lambda_{s}$, respectively, and the selection of the cutoff wavelength $\lambda_{x}$ depends on the compositions of the profiles. If the profile consists of the form error profile, waviness profile, and roughness profile, we select $\lambda_{s}$ as the cutoff wavelength $\lambda_{x}$, and if the profile includes the form error profile and waviness profile, $\lambda_{c}$ will be selected as $\lambda_{x}$. Otherwise, $\lambda_{f}$ should be selected as $\lambda_{x}$.

For the determination of the cutoff wavelength $\lambda_{x}$, we can first refer to the determining methods of the sampling length in the measurement of surface roughness to determine the parameter $\lambda_{s}$. The corresponding sampling length values of the surface roughness values $R a 0.008 \sim R a 2.0$ or $R z$ $0.025 \sim R z 10.0$ can be seen in Table 1 .

The object of the sampling length $l r$ stipulated in reference [8] is to eliminate the influence of waviness on the evaluation of surface roughness, so we may approximately take $l r$ as the cutoff wavelength $\lambda_{c 1}$. On the other hand, the usually adopted parameters of the Gaussian filter [9] in the roundness measurement are shown in Table 2. According to the relationship of the diameter of the measured cylindrical component and the cutoff wavelength of the Gaussian filter, we can gain the cutoff wavelength $\lambda_{c 2}$.

From Tables 1 and 2, it is known that $\lambda_{c 1}$ is related to the surface roughness value $R a$ or $R z$, and $\lambda_{c 2}$ depends on the diameter of the measured cylinder. Besides, the cutoff wavelengths of the form error profile, waviness profile, and roughness profile are usually classified as follows: that is, the profile with the wavelengths being equal to or greater than $10 \mathrm{~mm}$ belongs to the form error profile, the profile with the wavelengths less than $1 \mathrm{~mm}$ is the roughness profile, and the wavelengths of the waviness profile are equal to or greater than $1 \mathrm{~mm}$ and less than $10 \mathrm{~mm}$. Therefore, the cutoff wavelength $\lambda_{c 3}$ is $1 \mathrm{~mm}$. We can take one of $\lambda_{c 1}, \lambda_{c 2}$, and $\lambda_{c 3}$ as $\lambda_{c}$ based on the need of the evaluation item.

According to Nyquist sampling criterion [10], if a longer sampling interval than the Nyquist criterion is specified, the digitized signal suffers from aliasing distortion; therefore, more than 2 points are needed for each wave. For reducing aliasing distortion induced by digitization, the sampled points for each wave with a wavelength of $\lambda_{x}$ are selected as 4 , so the sampling interval in each roundness profile is as follows:

$$
\Delta s=\frac{\lambda_{x}}{4}
$$

where $\lambda_{x}$ may be $\lambda_{c}$ or $\lambda_{s}$. The total sampling number of each roundness profile can be gained by the following equation:

$$
n_{r}=\frac{\pi D}{\Delta s},
$$

and the angular interval $\Delta_{\mathrm{s}}=\Delta \psi_{r}=2 \pi / n_{r}$.

Suppose that the cutoff wavelength $\lambda_{f}$ of a generatrix is equal to $10 \lambda_{c}$ or $10 \mathrm{~mm}$ or larger based on the cylindrical 

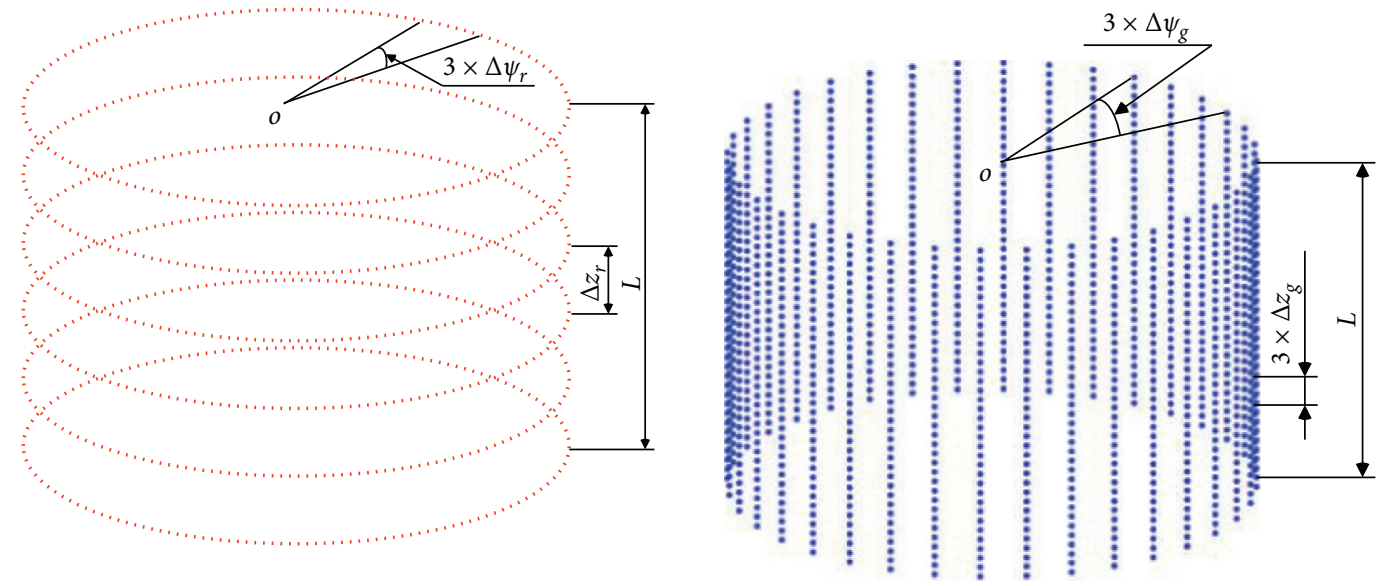

(a)

(b)
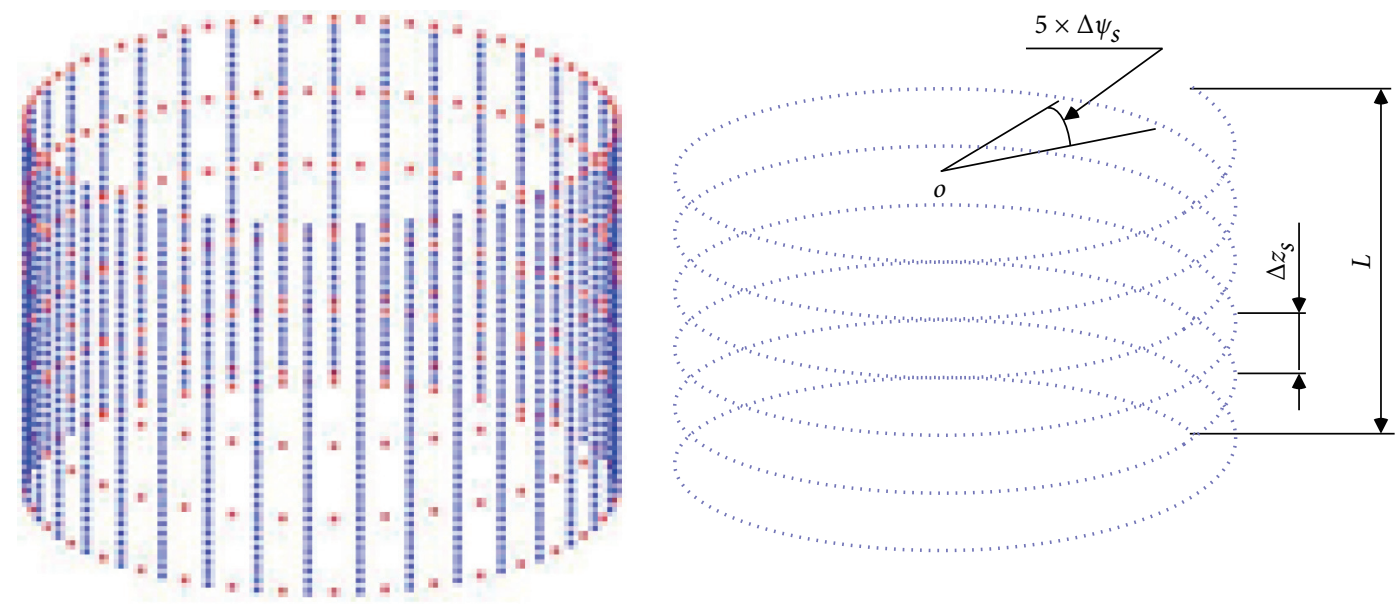

(c)

(d)

FIGURE 8: Schematic of parameters of the extraction strategies [6, 7]. (a) Roundness profile (RP). (b) Generatrix profile (GP). (c) Birdcage profile (BP). (d) Single spiral profile (SP).

TABLE 1: The relationship of surface roughness values and sampling length values [8].

\begin{tabular}{lcc}
\hline$R a(\mu \mathrm{m})$ & $R z(\mu \mathrm{m})$ & $\operatorname{lr}(\mathrm{mm})$ \\
\hline$\geq 0.008 \sim 0.02$ & $\geq 0.025 \sim 0.10$ & 0.08 \\
$>0.02 \sim 0.1$ & $>0.10 \sim 0.50$ & 0.25 \\
$>0.1 \sim 2.0$ & $>0.50 \sim 10.0$ & 0.8 \\
\hline
\end{tabular}

component's size or form tolerance and cylinder's length $L$, which does not consider the influence of waviness and roughness profiles, the interval $\Delta z_{r}$ of two adjacent roundness profiles may be equal to $\lambda_{f} / 4 \sim \lambda_{f} / 2$.

The parameters $\Delta \psi_{s}$ and $\Delta z_{s}$ of a single spiral profile in Figure $8(\mathrm{~d})$ may be equal to the parameters $\Delta \psi_{r}$ and $\Delta z_{r}$, respectively. The axial interval $\Delta z_{1}$ of two adjacent points in the axial direction is as follows:

$$
\Delta z_{1}=\frac{\Delta z_{s}}{n_{s}} .
$$

In equations $(1) \sim(7), z=(i-1) \Delta z_{1}$ and $\psi_{x}=(j-1) \Delta \psi_{x}$, where $x$ means $r$ for roundness profiles and $s$ for the single spiral profile. The number of the roundness profiles can be calculated as follows:

$$
n_{z}=\frac{L}{\Delta z_{1}}+1
$$

The pitch number $m_{s}$ of the single profile is equal to $m_{r}-1$. For setting $G$ in equation (3) and $S$ in equation (12), the size tolerance of component's diameter and the usual parallelism specification of the slideway and the spindle rotary axis can be considered. Suppose that the size tolerance of the component is $T_{s}$, the parallelism index of the slideway and the spindle rotary axis is $T_{p}$ and $C_{p k}=\eta$, and then size distribution $T_{6 \sigma}=T_{s} / \eta$. We can approximately assign one fourth of $T_{6 \sigma}$ to $G$ and $S$, respectively. For $g_{f w j}\left(j=1 \sim N_{g f}\right)$ in equation (2) and $r_{f w j}\left(j=1 \sim N_{r f}\right)$ in equation (9), both $g_{f w j}$ $\left(j=N_{g f}\right)$ and $r_{f w j}\left(j=N_{r f}\right)$ may be set as a quarter of the roundness tolerance or the cylindricity tolerance, and the values of $g_{f w j}\left(j=\left(N_{g f}-1 \sim 1\right)\right.$ or $r_{f w j}\left(j=\left(N_{r f}-1 \sim 1\right)\right.$ reduce in turn based on a priority number system $R 10$ or others. For $g_{r w j}\left(j=1 \sim N_{g r}\right)$ in equation (5) and $r_{r w j}\left(j=1 \sim N_{r r}\right)$ in equation (11), both $g_{r w j}\left(j=N_{g r}\right)$ and $r_{r w j}\left(j=N_{r r}\right)$ may be set as surface roughness $R a$, and the values of $g_{r w j}\left(j=\left(N_{g r}-1 \sim 1\right)\right.$ 
TABLE 2: The relationship of the diameter of the measured component and Gaussian filter's cutoff wavelength.

\begin{tabular}{|c|c|c|c|c|c|}
\hline $\begin{array}{l}\text { Diameter of component } \\
(\mathrm{mm})\end{array}$ & $\begin{array}{l}\text { Filter's cutoff } \\
\text { (UPR) }\end{array}$ & $\begin{array}{l}\text { Wavelength } \lambda_{c 2} \\
(\mathrm{~mm})\end{array}$ & $\begin{array}{l}\text { Diameter of component } \\
(\mathrm{mm})\end{array}$ & $\begin{array}{l}\text { Filter's cutoff } \\
\text { (UPR) }\end{array}$ & $\begin{array}{l}\text { Wavelength } \lambda_{c 2} \\
(\mathrm{~mm})\end{array}$ \\
\hline$\leq 8$ & 15 & $\leq 1.675$ & $>80 \sim 250$ & 500 & $>1.675 \sim 1.57$ \\
\hline$>8 \sim 25$ & 50 & $>1.675 \sim 1.57$ & $>250$ & 1500 & $>0.523$ \\
\hline$>25 \sim 80$ & 150 & $>1.57 \sim 1.675$ & & & \\
\hline
\end{tabular}

or $r_{r w j}\left(j=\left(N_{r r}-1 \sim 1\right)\right.$ reduce in turn based on a priority number system $R 10$. For $g_{w w j}\left(j=1 \sim N_{\mathrm{gw}}\right)$ in equation (4) and $r_{\mathrm{wwj}}\left(j=1 \sim N_{\mathrm{rw}}\right)$ in equation (10), both $g_{w w j}\left(j=N_{g w}\right)$ and $r_{w w j}\left(j=N_{r w}\right)$ may be set as two times of the surface roughness $R a$, and the values of $g_{w w j}\left(j=\left(N_{g w}-1 \sim 1\right)\right.$ or $r_{w w j}\left(j=\left(N_{r w}-1 \sim 1\right)\right.$ reduce in turn based on a priority number system $R 10$.

For the initial angles in equations (2), (4), (5), and (9)-(11), they may not abide by any rule and may be set according to random probability distribution in a range from zero to $2 \pi$.

Considering that the radial cutting force of the precision manufacturing is not large, the influence of the bending deformation caused by the radial cutting force on the simulation of cylinder's size feature is not considered in this paper. On the other hand, even if the roughness profile in equations (1) and (6) is all included, owing to that only cylinder's size feature needs to be simulated, the simulation of cylinder's size feature can eliminate the roughness profile.

\section{Simulation Experiment and Discussion}

4.1. Simulation Experiment. We took a shaft in Figure 9 as an example for the extraction simulation of the cylinder's size feature based on the models in Sections 2 and 3. The diameter and its tolerance, upper limit deviation, roundness tolerance, cylindricity tolerance, surface roughness $R a$, and length of the shaft are shown in Figure 9. Four extraction strategies of cylinder's feature in Figure 8 were adopted for the cylindrical component in Figure 9. The simulation parameters are shown in Table 3, where $X$ can be replaced by the symbols 0,1 , and 2 , respectively. The profiles indicated by the symbol 0 consist of the form, waviness, and roughness profiles, the profiles indicated by the symbol 1 include the form and waviness profiles, and the profiles indicated by the symbol 2 are only the form profiles. In Table 3, tilt_angle is the angle between the axis of the simulated cylinder and the $z$ axis. The cylindrical features shown in Figures 10-17 are simulated according to the parameters in Table 3, and the sampling number of which is 2000, where RP, GP, BP, and $\mathrm{SP}$ represent the profiles gained based on the extraction strategies of the roundness profiles, generatrix profiles, birdcage profile, and single spiral profile, respectively.

4.2. Evaluation of Cylindrical Features. The evaluation objects of cylindrical features include global sizes, calculated sizes, form errors, waviness, and roughness in this paper. The evaluation results of global sizes and cylindricity errors based on the cylindrical features simulated by using the parameters in Table 3 and the evaluation models [5] are shown in Figures 18 and 19, where LSD, MCD, MID, and

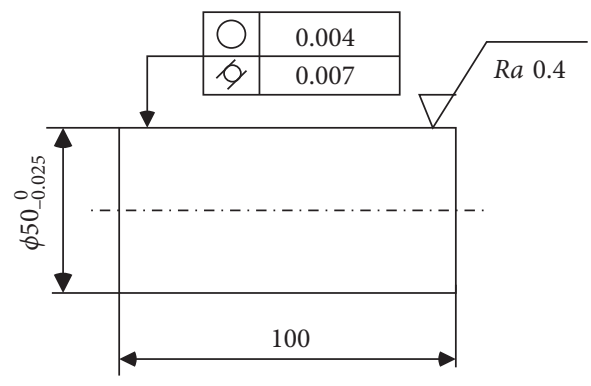

FIgURE 9: Cylindrical component used for simulation of feature extraction.

MMD are the abbreviations of least-squares diameters, minimum circumscribed diameters, maximum inscribed diameters, and minimax diameters, respectively, and LSC, MCC, MIC, and MZC are the abbreviations of cylindricity errors evaluated by least-square criterion, minimum circumscribed criterion, maximum inscribed criterion, and minimum zone criterion [11-14], respectively. The evaluated roundness errors of the cylindrical features based on the roundness profile extraction strategy and the corresponding models $[15,16]$ are shown in Figure 20, where MEAN, MAX, and MIN represent the mean, maximum, and minimum values of roundness errors for one cylindrical feature, respectively, and their corresponding calculated size is shown in Figure 21, where C_D, A_D, and V_D represent the circumference diameter, area diameter, and volume diameter of the calculated sizes, which were evaluated based on the corresponding roundness profiles above and the models [17], and the meanings of MEAN, MAX, and MIN can be seen in Figure 20.

For the evaluation of the axis straightness error of a cylinder, the center $o_{i}\left(a_{i}, b_{i}, z_{i}\right)$ of the reference circle of the $i$ th roundness profiles is first determined, as shown in Figure 22, where $\rho_{i j}$ is the discretized value of $\rho(z, \psi(z))$ in equation (13), that is, the radial distance from the $j$ th sampling point in the $i$ th roundness profile to the rotary axis, $\psi_{i j}$ is the angle between the $j$ th sampling point in the $i$ th roundness profile and the $x$ axis, and $e_{i}$ and $\varepsilon_{i}$ are the eccentricity and eccentric angles of the $i$ th reference circle. The reference circle may be the least-square reference cycle or the minimum circumscribed reference circle or the maximum inscribed reference circle or the minimum zone reference circle, which corresponds to four kinds of evaluation criteria of the roundness error mentioned above.

The actual axis of the simulated cylinder can be expressed as the sequential connection of centers of the reference circles, as shown in Figure 23. 
TABLE 3: Simulation parameters of cylindrical features of the part in Figure 9.

\begin{tabular}{|c|c|c|c|c|c|c|c|c|c|}
\hline Symbol & 1N1_X & 1N3_X & 3N1_X & 3N3_X & 1Y1_X & $1 Y 3 \_X$ & 3Y1_X & 3Y3_X & Remarks \\
\hline$G(\mathrm{~mm})$ & 0 & 0 & 0.001 & 0.001 & 0 & 0 & 0.001 & 0.001 & \multirow{2}{*}{ Equation (3) } \\
\hline$k_{q}$ & 0 & 0 & 3 & 3 & 0 & 0 & 3 & 3 & \\
\hline$N_{g,}, N_{g w}, N_{g r}$ & \multicolumn{8}{|c|}{5} & \multirow{3}{*}{ Equations (2), (4), (5) } \\
\hline$g_{f w j}, g_{w w j}, g_{r w j}$ & \multirow{2}{*}{\multicolumn{8}{|c|}{$\begin{array}{c}g_{f w 1} \sim g_{f w 5}=0.112 \sim 0.560 \mu \mathrm{m} ; g_{w w 1} \sim g_{w w 5}=0.066 \sim 0.320 \mu \mathrm{m} ; \\
g_{r w 1} \sim g_{r w 5}=0.050 \sim 0.250 \mu \mathrm{m} \\
\text { Random values between } 0 \text { and } 2 \pi\end{array}$}} & \\
\hline$\theta_{j}, \varphi_{j}, \vartheta_{j}$ & & & & & & & & & \\
\hline$e(0)(\mathrm{mm})$ & \multirow{2}{*}{\multicolumn{8}{|c|}{$\begin{array}{l}0.001 \\
\text { Random values from between } 0 \text { and } 2 \pi\end{array}$}} & \multirow{2}{*}{ Equation (7) } \\
\hline$\phi(z)$ & & & & & & & & & \\
\hline$R(\mathrm{~mm})$ & \multicolumn{8}{|c|}{$(50-0.025) / 2$} & \multirow{3}{*}{ Equation (8) } \\
\hline$A(\mathrm{~mm})$ & 0 & 0.0005 & 0 & 0.0005 & 0 & 0.0005 & 0 & 0.0005 & \\
\hline$k_{r}$ & 1 & 3 & 1 & 3 & 1 & 3 & 1 & 3 & \\
\hline$N_{r f}, N r_{w}, N_{r r}$ & \multicolumn{8}{|c|}{5} & \multirow{3}{*}{ Equations (9)-(11) } \\
\hline$r_{f w j}, r_{w w j}, r_{r w j}$ & \multirow{2}{*}{\multicolumn{8}{|c|}{$\begin{array}{c}r_{f w 1} \sim r_{f w 5}=0.112 \sim 0.560 \mu \mathrm{m} ; r_{w w 1} \sim r_{w w 5}=0.066 \sim 0.320 \mu \mathrm{m} ; \\
r_{r w 1} \sim r_{r w 5}=0.050 \sim 0.250 \mu \mathrm{m} \\
\text { Random values between } 0 \text { and } 2 \pi\end{array}$}} & \\
\hline$\theta_{j}, \varphi_{j}, \vartheta_{j}$ & & & & & & & & & \\
\hline$\lambda_{f}, \lambda_{c}, \lambda_{s}$ & \multicolumn{8}{|c|}{$\lambda_{f}=1.6225 \mathrm{~mm} ; \lambda_{c}=0.3 \mathrm{~mm}, \lambda_{s}=0.06 \mathrm{~mm}$} & Equations (2), (4), (5), (9)-(11) \\
\hline$\delta(z), \delta(\psi(z))(\mathrm{mm})$ & \multicolumn{8}{|c|}{ Random values between 0 and $0.5 * g_{r w 1}$ or between 0 and $0.5 * r_{r w 1}$} & Equations (5), (11) \\
\hline$S$ & 0 & 0 & 0 & 0 & 0.001 & 0.001 & 0.001 & 0.001 & \multirow{7}{*}{ Equation (13) } \\
\hline$C D(\mathrm{~mm})$ & \multicolumn{8}{|r|}{ 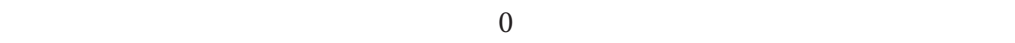 } & \\
\hline$L(\mathrm{~mm})$ & \multirow{2}{*}{\multicolumn{8}{|c|}{$\begin{array}{c}100 \\
0.001\end{array}$}} & \\
\hline tilt_angle (radian) & & & & & & & & & \\
\hline$m_{r}, m_{s}$ & \multirow{2}{*}{\multicolumn{8}{|c|}{$\begin{array}{c}m_{r}=L / 2.5+1=41, m_{s}=L / 2.5=40 \\
500,625,1000,1250,2000,2500,5000 \text {, and } 10000 \text { in the same extraction sample }\end{array}$}} & \\
\hline$n_{r}, n_{s}$ & & & & & & & & & \\
\hline$n_{g}, m_{g}$ & \multicolumn{8}{|c|}{$n_{g}=50 \pi / 2.5 \approx 63, m_{g}=m_{r} * n_{r} / n_{g} \approx 1302$} & \\
\hline
\end{tabular}

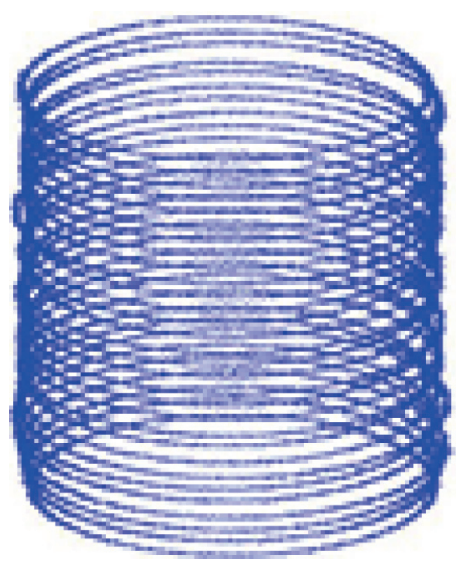

(a)

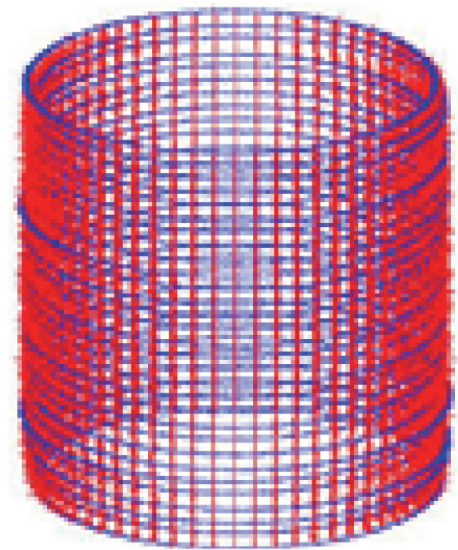

(c)

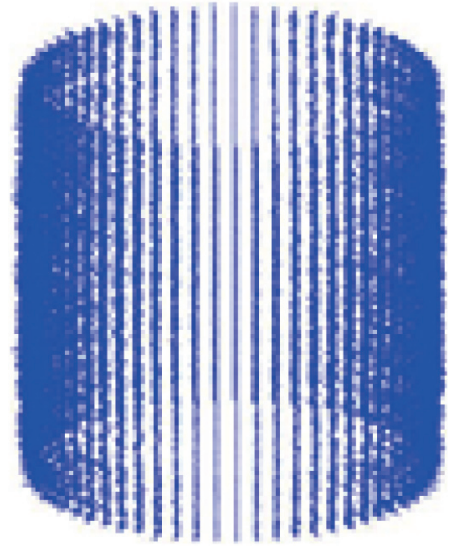

(b)

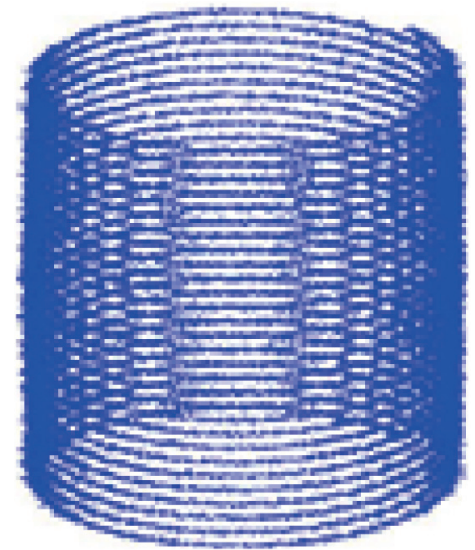

(d)

FIgURE 10: Cylindrical features gained based on the simulation parameter symbol “1N1_0.” (a) RP. (b) GP. (c) BP. (d) SP. 


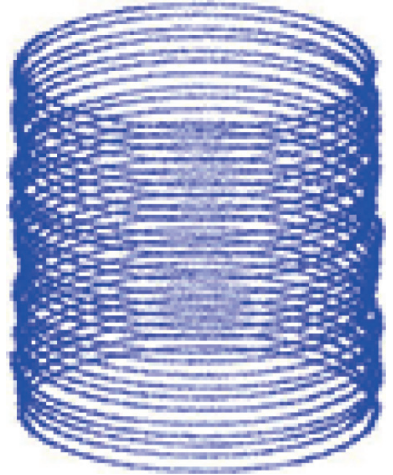

(a)

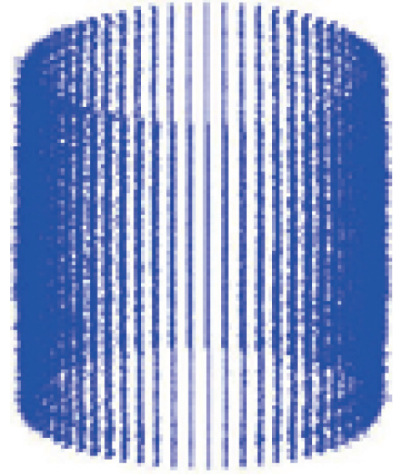

(b)

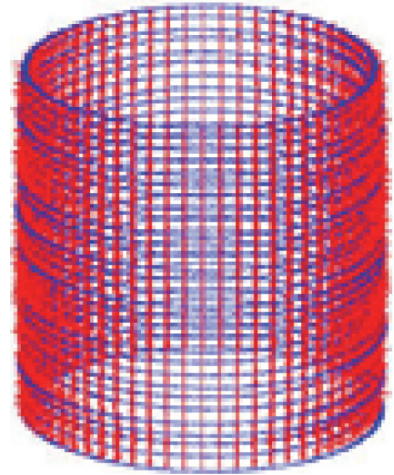

(c)

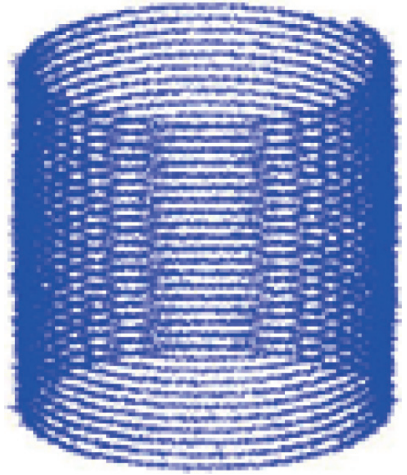

(d)

FIgURE 11: Cylindrical features gained based on the simulation parameter symbol “1N3_0." (a) RP. (b) GP. (c) BP. (d) SP.

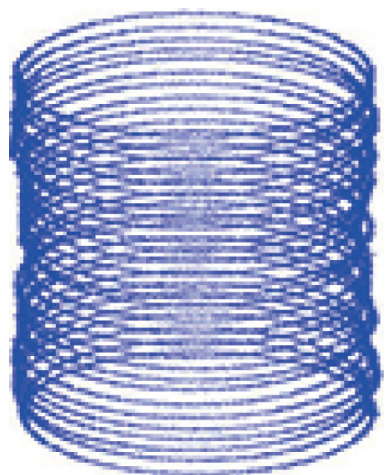

(a)

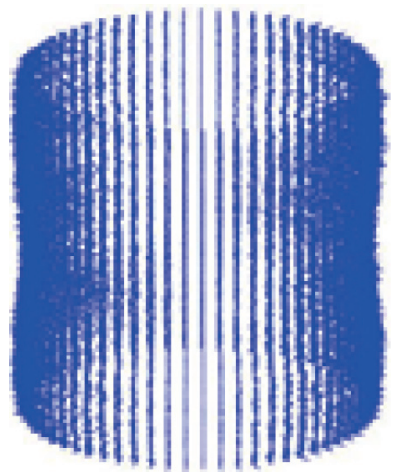

(b)

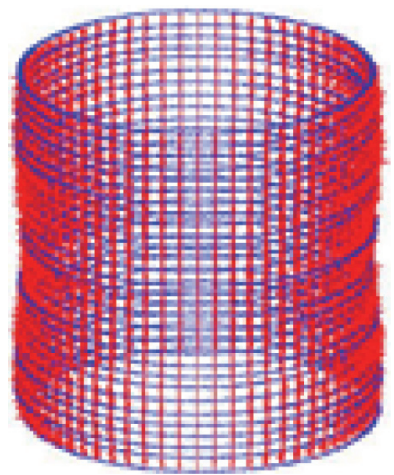

(c)

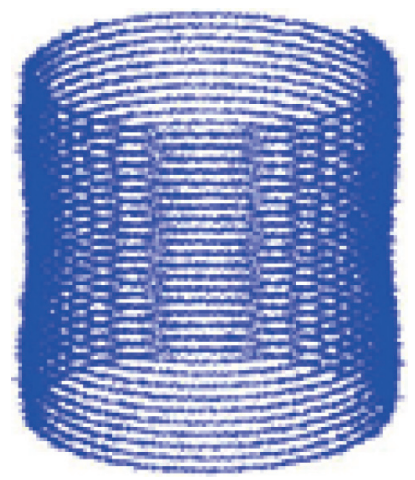

(d)

FIgURE 12: Cylindrical features gained based on the simulation parameter symbol "3N1_0." (a) RP. (b) GP. (c) BP. (d) SP.

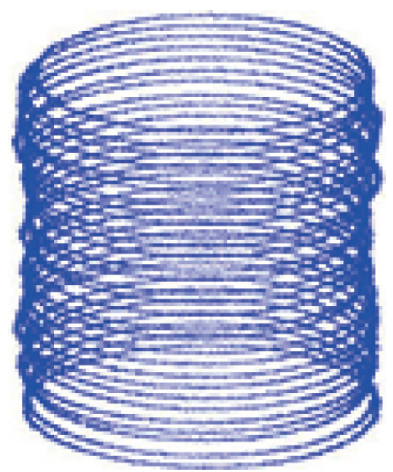

(a)

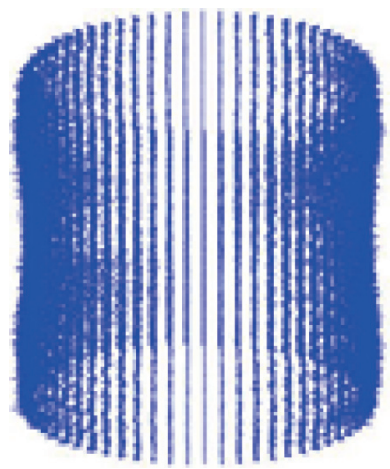

(b)

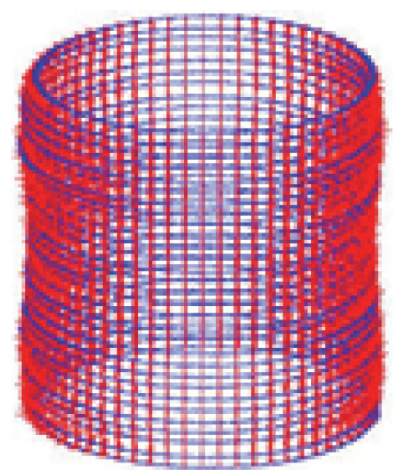

(c)

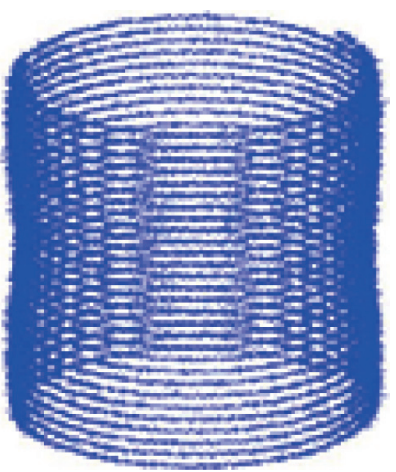

(d)

Figure 13: Cylindrical features gained based on the simulation parameter symbol "3N3_0.” (a) RP. (b) GP. (c) BP. (d) SP.

There are two evaluation criterions of the axis straightness error, that is, least-square criterion and minimum zone criterion. Suppose that the ideal axis in Figure 23 is characterized by the parameters $x_{a 0}, y_{a 0}, p_{a}$, and $q_{a}$, the evaluation of the axis straightness based on the least-square criterion should meet the requirement as follows:

$$
F_{a}=\sum_{i=1}^{m_{r}} d_{r i}^{2}=\min
$$

where $d_{r i}$ is the radial distance from the center $o_{i}$ of the $i$ th reference circle to the ideal axis, as shown in Figure 23, which can be expressed as follows:

$$
d_{r i}=\sqrt{\left(a_{i}-x_{a 0}-p_{a} z_{i}\right)^{2}+\left(b_{i}-y_{a 0}-q_{a} z_{i}\right)^{2}}
$$

where the parameters $x_{a 0}, y_{a 0}, p_{a}$, and $q_{a}$ of the ideal axis are undetermined ones. 


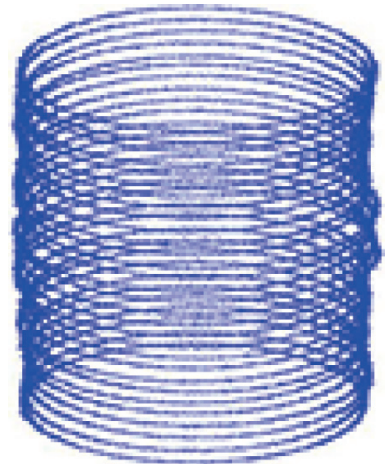

(a)

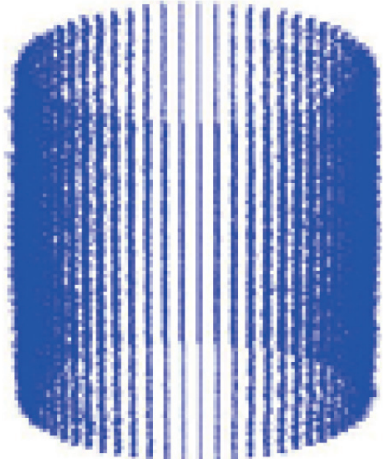

(b)

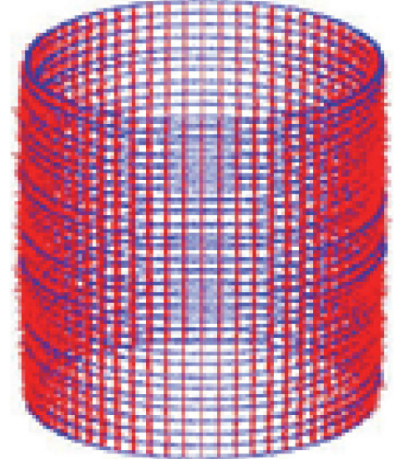

(c)

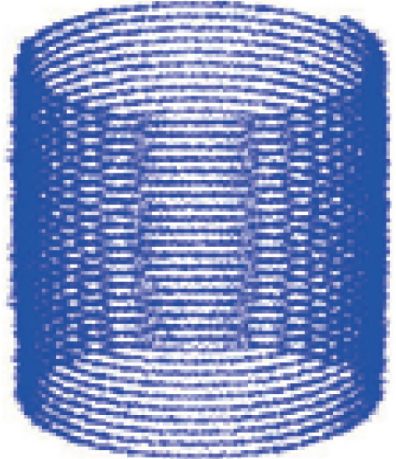

(d)

FIgURE 14: Cylindrical features gained based on the simulation parameter symbol "1Y1_0.” (a) RP. (b) GP. (c) BP. (d) SP.

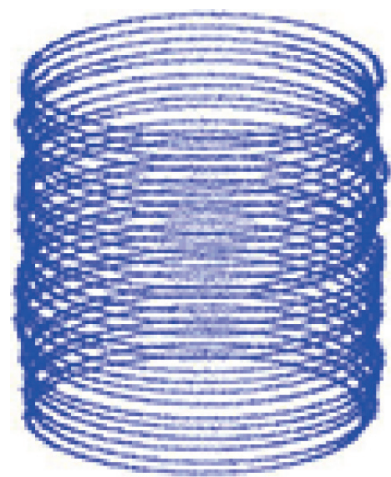

(a)

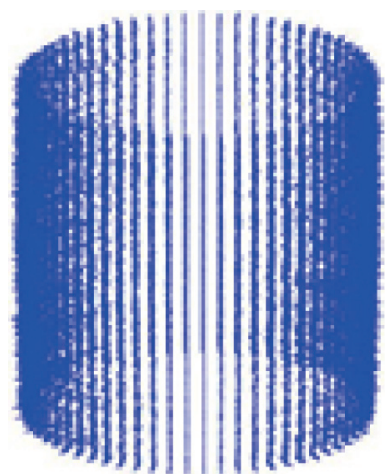

(b)

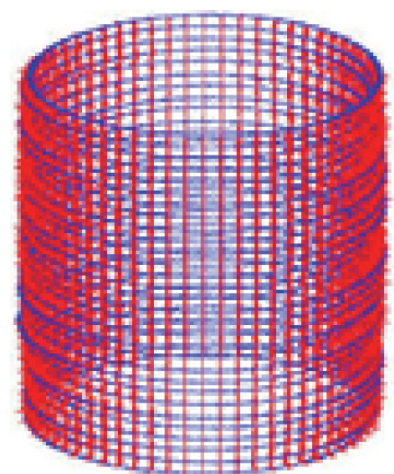

(c)

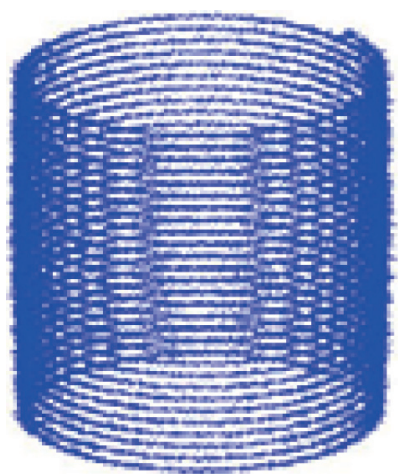

(d)

Figure 15: Cylindrical features gained based on the simulation parameter symbol "1Y3_0.” (a) RP. (b) GP. (c) BP. (d) SP.

Let the partial derivatives of $F_{a}$ with respect to variables $x_{a 0}, y_{a 0}, p_{a}$, and $q_{a}$ be equal to zero, respectively, and a linear system of equations with variables $x_{a 0}, y_{a 0}, p_{a}$, and $q_{a}$ can be obtained. After solving the linear system of equations, the parameters $x_{a 0}, y_{a 0}, p_{a}$, and $q_{a}$ of the ideal axis in Figure 23 can be gained, and the axis straightness error $f_{a \mathrm{LS}}$ determined based on the least-square criterion can be expressed as follows:

$$
f_{a \mathrm{LS}}=2 \max _{1 \leq i \leq m_{r}} d_{v i}
$$

where $m_{r}$ is the number of the roundness profiles and $d_{v i}$ is the vertical distance from the center $o_{i}$ of the $i$ th reference circle to the ideal axis, which can be calculated by the following equation:

$$
d_{v i}=\sqrt{\left(a_{i}-x_{a 0}\right)^{2}+\left(b_{i}-y_{a 0}\right)^{2}+z_{i}^{2}-\frac{\left[p_{a 0}\left(a_{i}-x_{a 0}\right)+q_{a 0}\left(b_{i}-y_{a 0}\right)+z_{i}\right]^{2}}{1+p_{a}^{2}+q_{a}^{2}}} .
$$

Based on the definition of the minimum zone criterion, the axis straightness error $f_{a \mathrm{MZ}}$ can be expressed as follows:

$$
\begin{aligned}
f_{a \mathrm{MZ}} & =2 \min _{X_{a}} \max _{1 \leq i \leq m_{r}} d_{v i}, \\
X_{a} & =\left[\begin{array}{llll}
x_{a 0} & y_{a 0} & p_{a} & q_{a}
\end{array}\right]^{\mathrm{T}},
\end{aligned}
$$

where $X_{a}$ represents the parameters of the ideal axis, which may be obtained through the optimization algorithm, and the parameters of the ideal axis determined based on the least-square criterion are usually taken as their initial values of the parameters of the ideal axis determined by using the minimum zone criterion through optimization.

For the evaluation of the generatrix straightness errors of a cylinder, a $z o \rho_{j}$ coordinate system is built for the $j$ th generatrix profile, as shown in Figure 24, where $\rho_{j i}$ is the radial distance from the $i$ th sampling point in the $j$ th generatrix profile to the $z$ axis and $d_{j i}$ is the radial distance from the $i$ th sampling point in the $j$ th generatrix profile to 


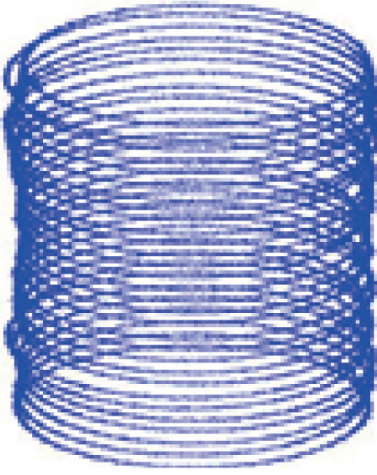

(a)

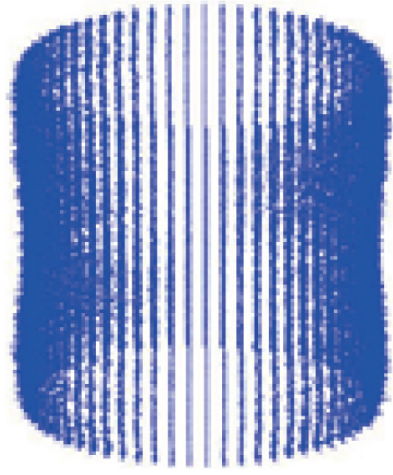

(b)

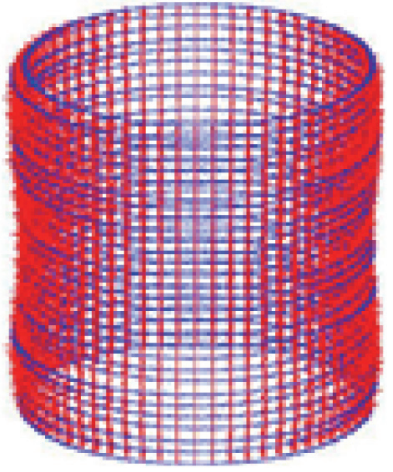

(c)

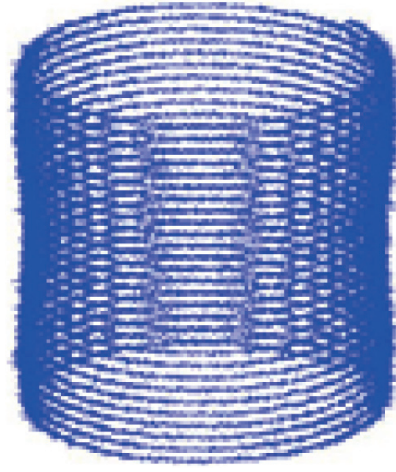

(d)

FIgURE 16: Cylindrical features gained based on the simulation parameter symbol "3Y1_0." (a) RP. (b) GP. (c) BP. (d) SP.

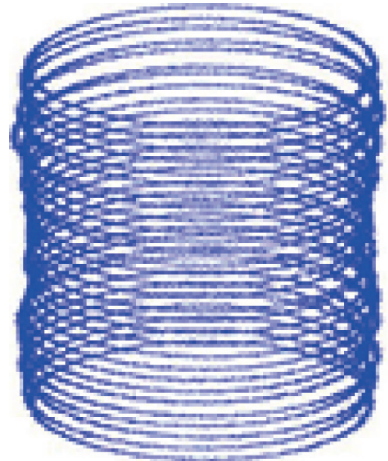

(a)

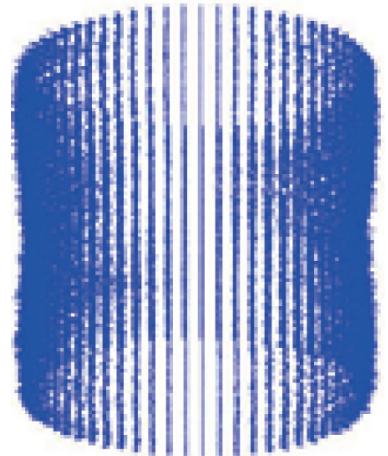

(b)

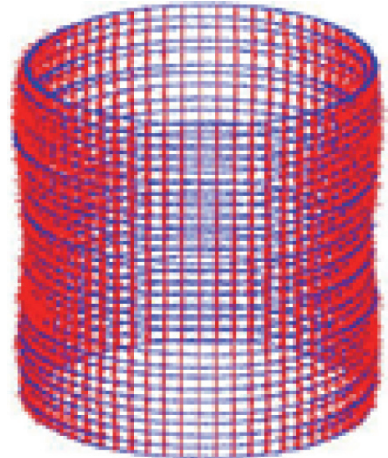

(c)

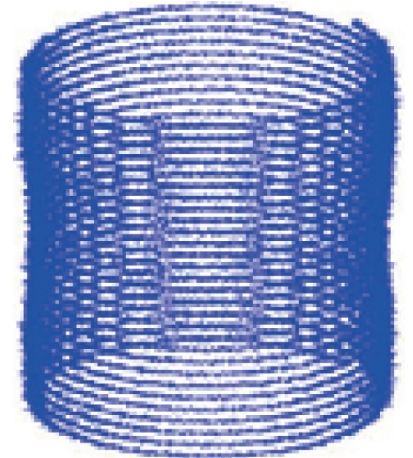

(d)

FIGURE 17: Cylindrical features gained based on the simulation parameter symbol "3Y3_0.” (a) RP. (b) GP. (c) BP. (d) SP.

the ideal line, which may be determined by using the leastsquare criterion or the minimum zone criterion.

Suppose that the slope and the intercept of the ideal line are $A_{i}$ and $B_{j}$, and then the ideal line in Figure 24 can be expressed as follows:

$$
\rho_{j}=A_{j} z_{i}+B_{j} .
$$

The least-square ideal line should satisfy the following condition; that is,

$$
F_{g}=\sum_{i=1}^{m_{g}} d_{j i}^{2}=\sum_{i=1}^{m_{g}}\left(\rho_{j i}-A_{j} z_{i}-B_{j}\right)^{2}=\min
$$

where $m_{g}$ is the number of the sampling points in the $j$ th generatrix profile.

Let the partial derivatives of $F_{g}$ with respect to variables $A_{j}$ and $B_{j}$ be equal to zero, respectively, and a linear system of equations with variables $A_{j}$ and $B_{j}$ can be obtained. After solving the linear system of equations, the parameters $A_{j}$ and $B_{j}$ of the ideal line in Figure 24 can be gained, and the generatrix straightness error $f_{g L S j}$ of the $j$ th generatrix profile by using the least-square criterion can be expressed as follows:

$$
f_{g L S j}=\frac{1}{\sqrt{1+A_{j}^{2}}}\left\{\max _{1 \leq i \leq m_{g}} d_{j i}-\min _{1 \leq i \leq m_{g}} d_{j i}\right\} .
$$

According to the definition of the minimum zone method, the generatrix straightness error $f_{g M Z j}$ can be expressed as follows:

$$
f_{g M Z j}=\frac{1}{\sqrt{1+A_{j}^{2}}} \min _{A_{j}, B_{j}}\left\{\max _{1 \leq i \leq m_{g}} d_{j i}-\min _{1 \leq i \leq m_{g}} d_{j i}\right\} .
$$

Through optimizing the parameters $A_{j}$ and $B_{j}$ of the ideal line and realizing the object function in equation (26), the minimum zone generatrix straightness error can be obtained. The parameters of the least-square ideal axis are usually taken as their initial values of the parameters of the minimum zone ideal axis for optimization.

The evaluation results of axis straightness errors can be seen in Figure 25(a), where LS- $x \times$, MC- $x \times$, MI- $x \times$, and MZ$x \times$ represent the center coordinates of least-square reference circles, minimum circumscribed reference circles, maximum inscribed reference circles, and minimum zone reference circles for all roundness profiles of one cylindrical 


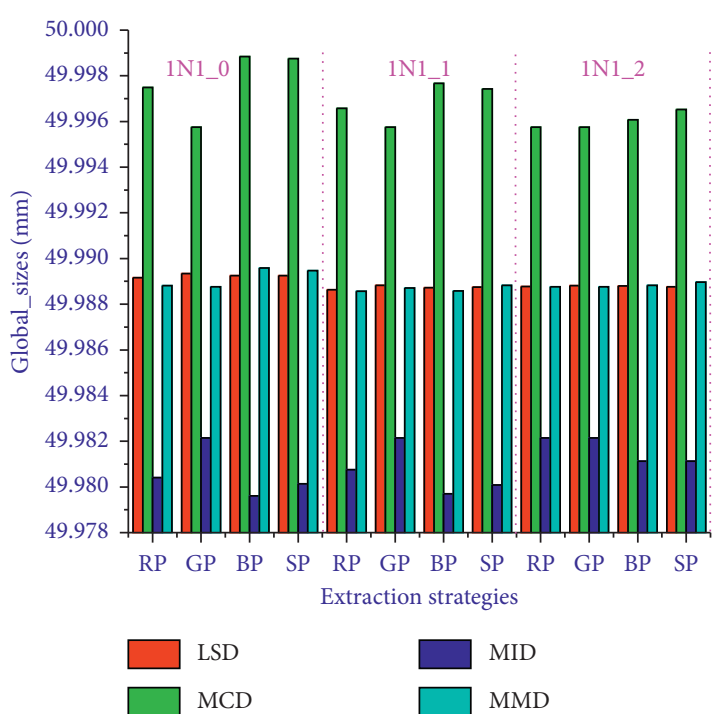

(a)
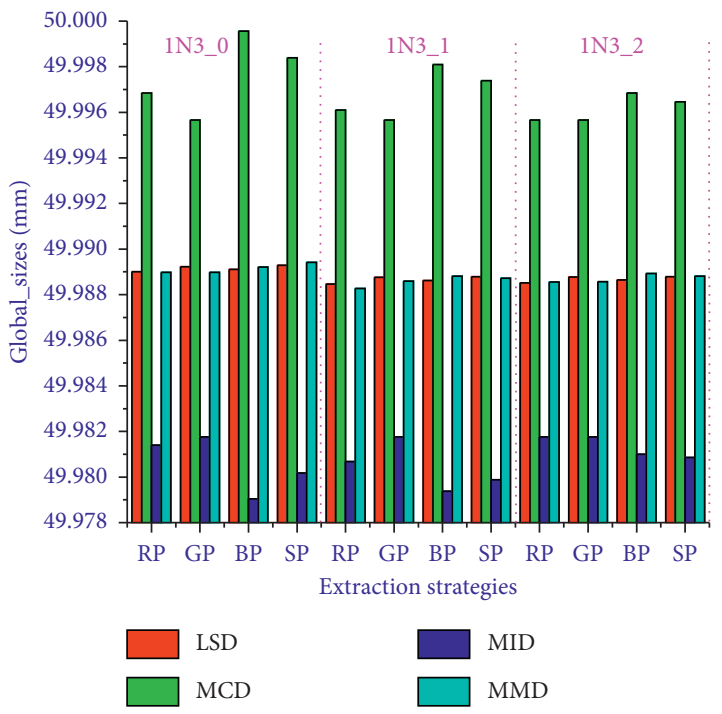

(c)

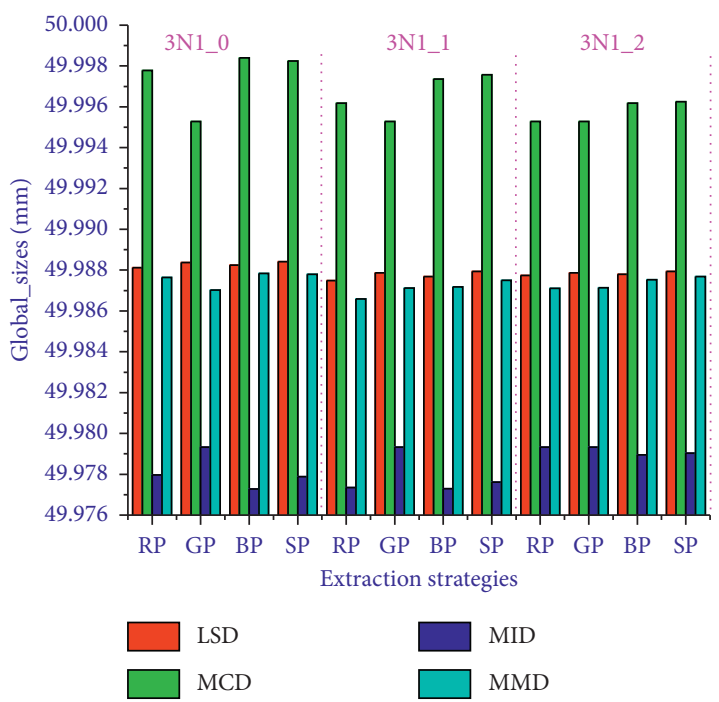

(e)

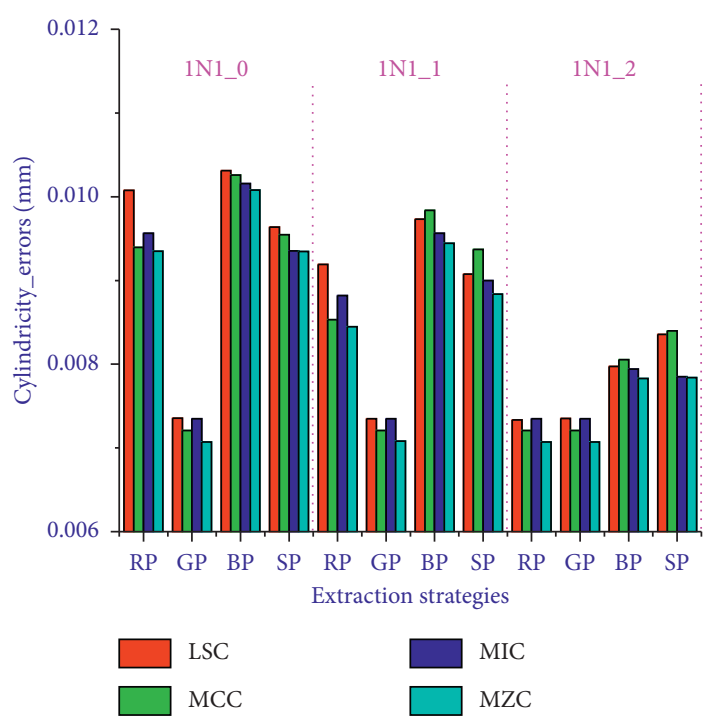

(b)

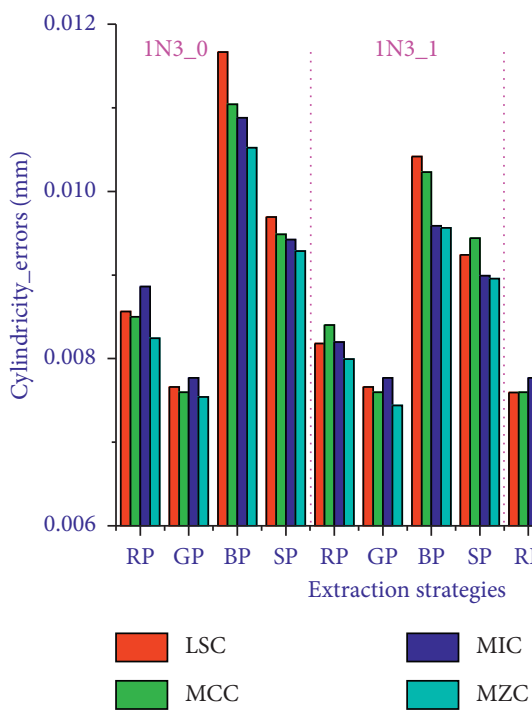

(d)

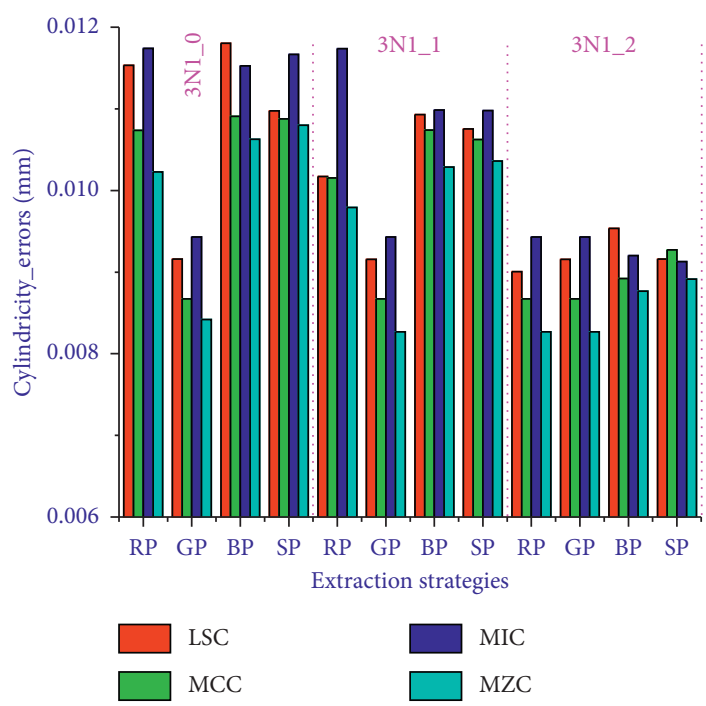

(f)

FIgURE 18: Continued. 


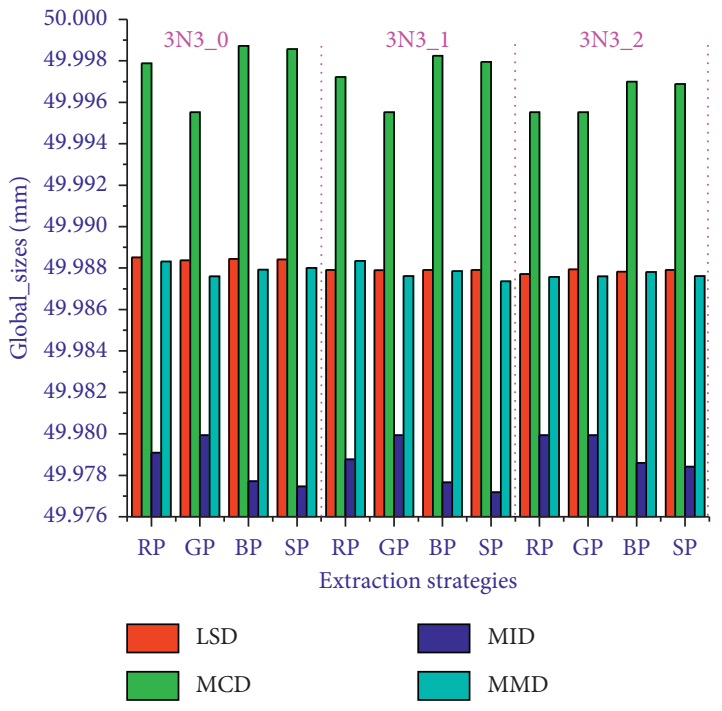

$(\mathrm{g})$

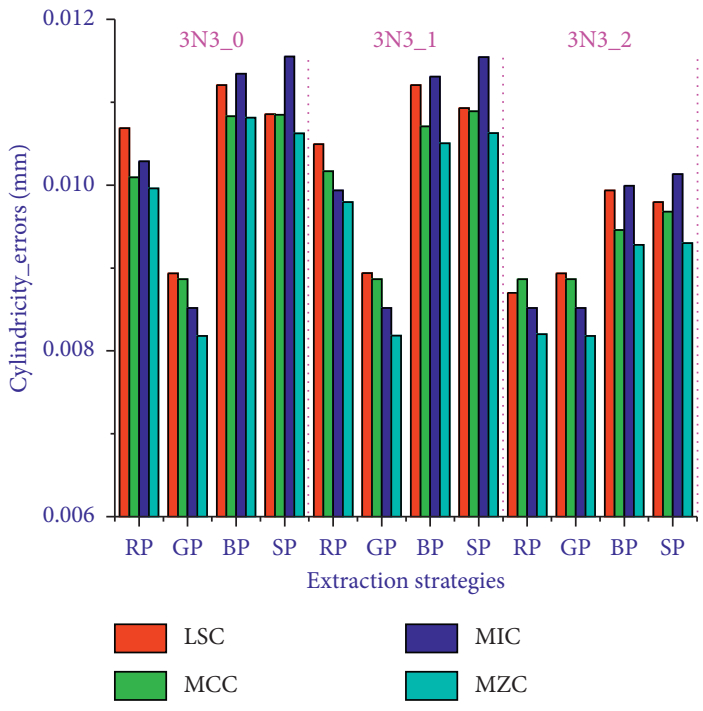

(h)

FIGURE 18: Global sizes and cylindricity errors of the cylindrical features produced under parameter symbols 1N1, 1N3, 3N1, and 3N3. (a) 1N1-global sizes. (b) 1N1-cylindricity errors. (c) 1N3-global sizes. (d) 1N3-cylindricity errors. (e) 3N1-global sizes. (f) 3N1-cylindricity errors. (g) 3N3-global sizes. (h) 3N3-cylindricity errors.

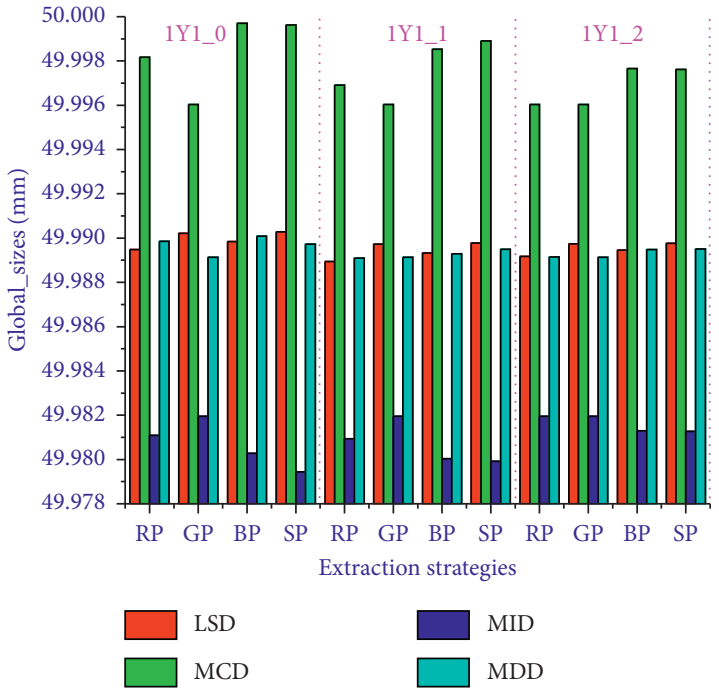

(a)

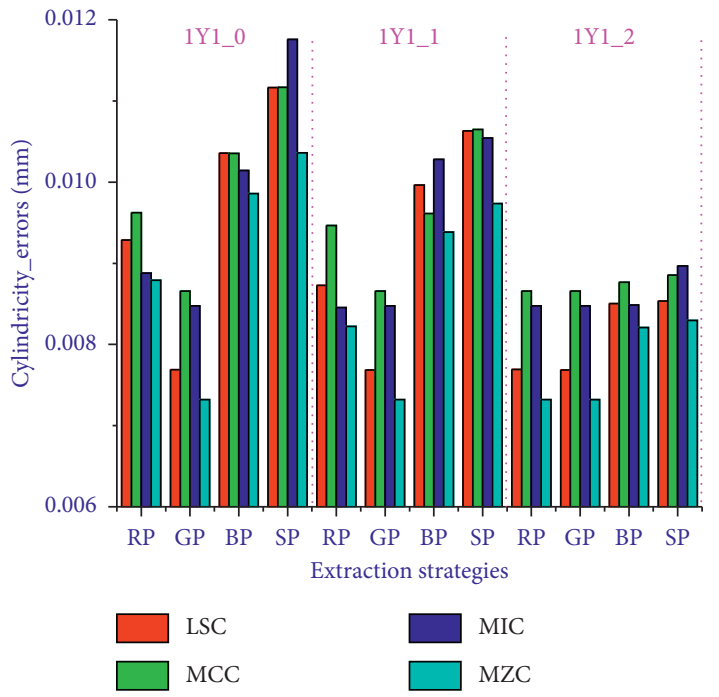

(b)

FIGURE 19: Continued. 


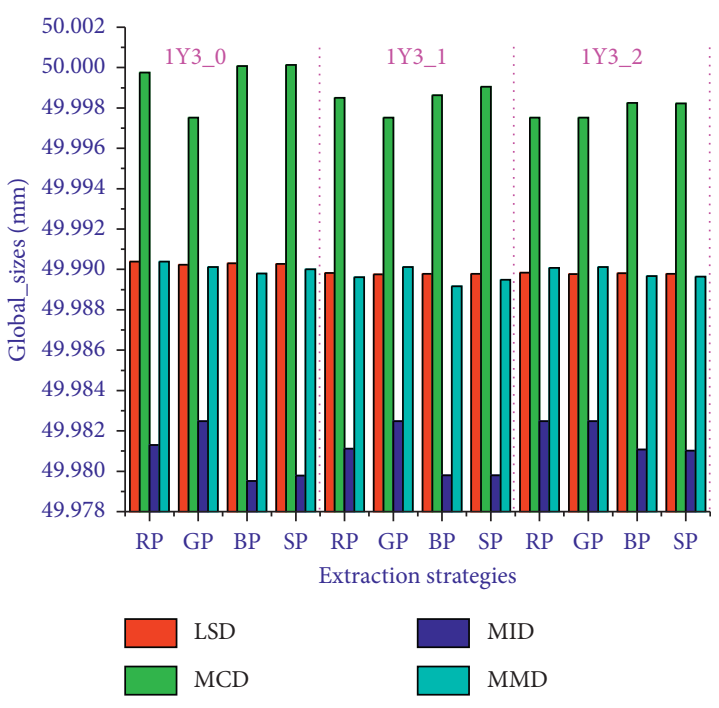

(c)
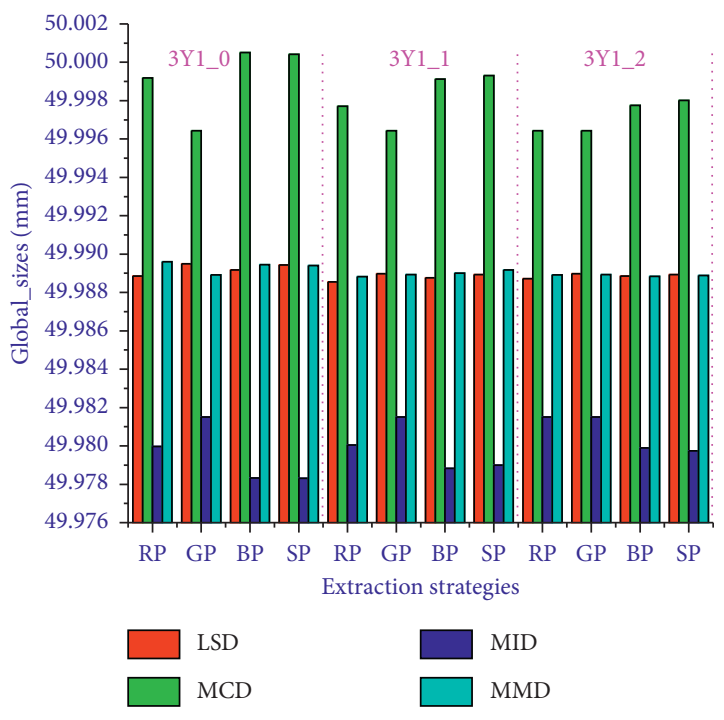

(e)
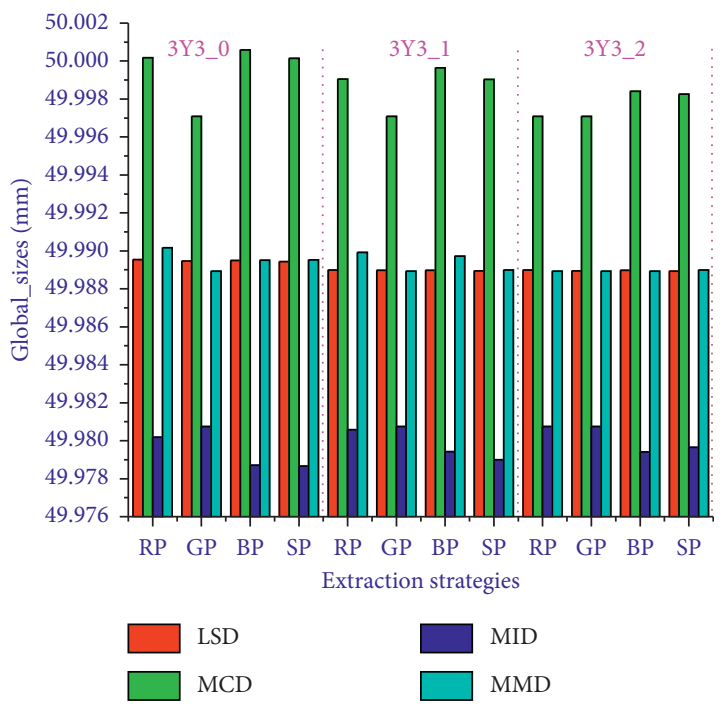

(g)

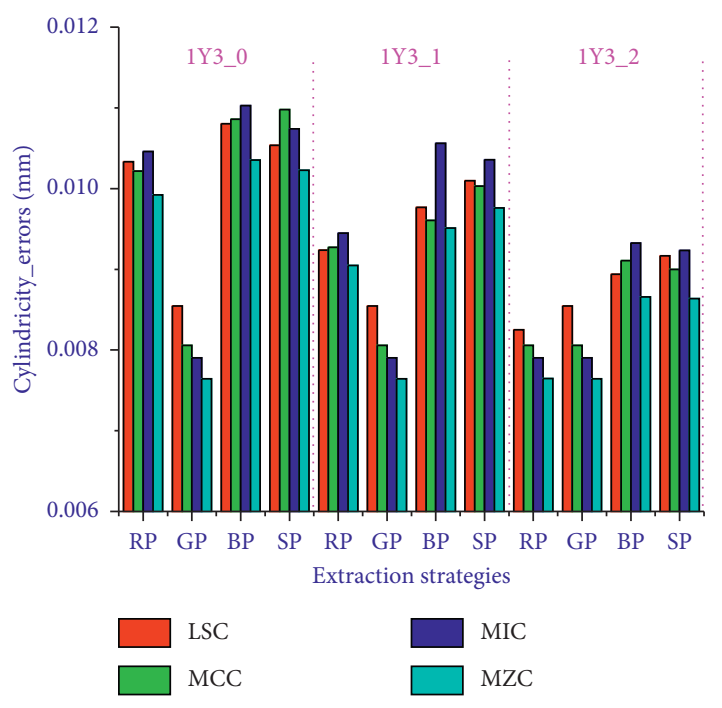

(d)

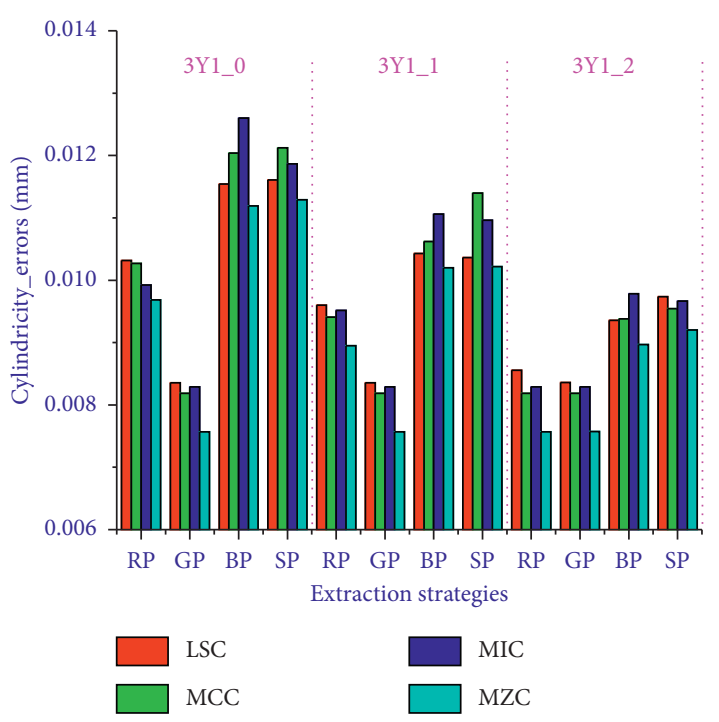

(f)

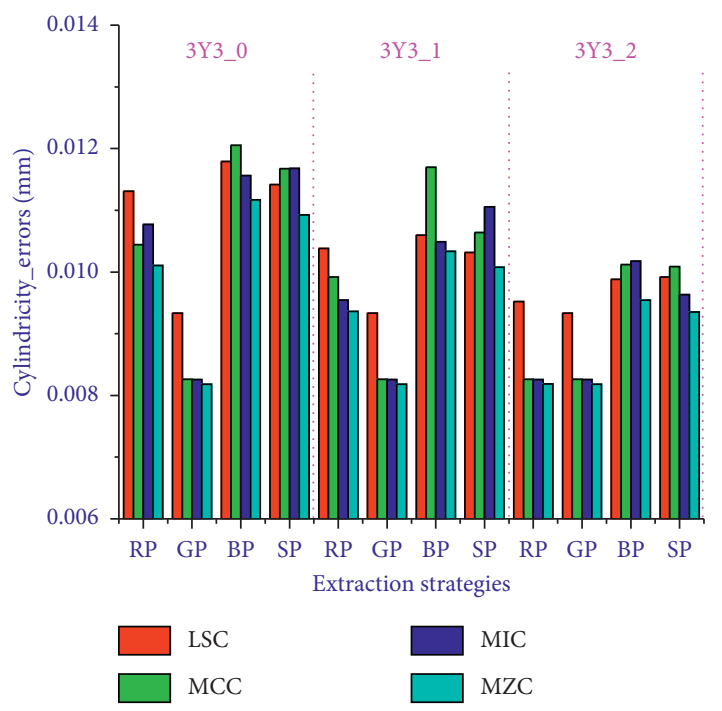

(h)

FIGURE 19: Global sizes and cylindricity errors of the cylindrical features produced under parameter symbols 1Y1, 1Y3, 3Y1, and 3Y3. (a) 1Y1-global sizes. (b) 1Y1-cylindricity errors. (c) 1Y3-global sizes. (d) 1Y3-cylindricity errors. (e) 3Y1-global sizes. (f) 3Y1-cylindricity errors. (g) 3Y3-global sizes. (h) 3Y3-cylindricity errors. 


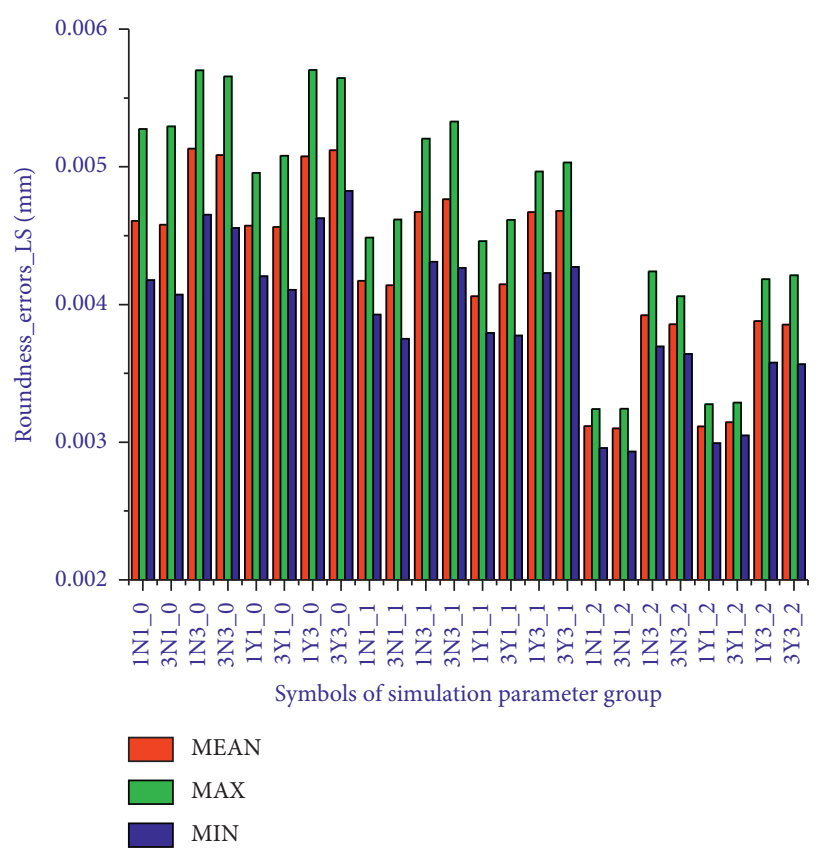

(a)

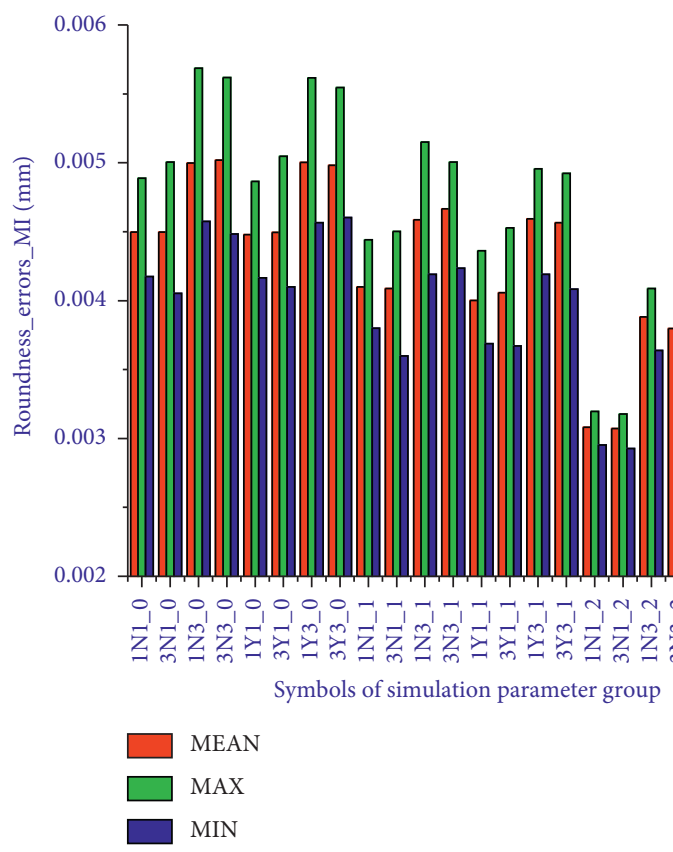

(c)

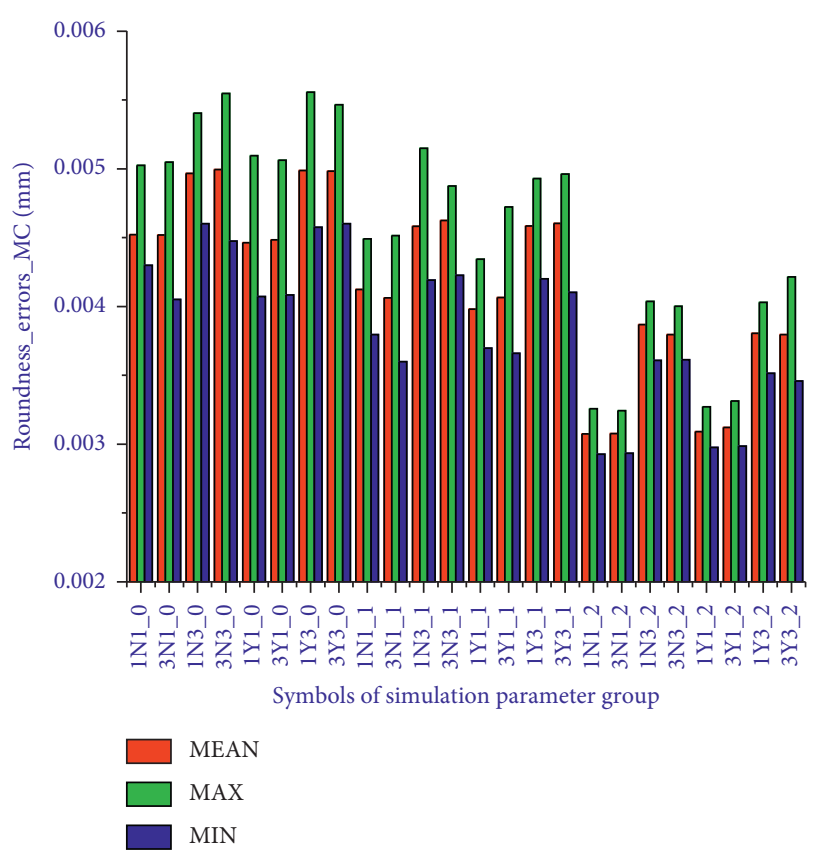

(b)

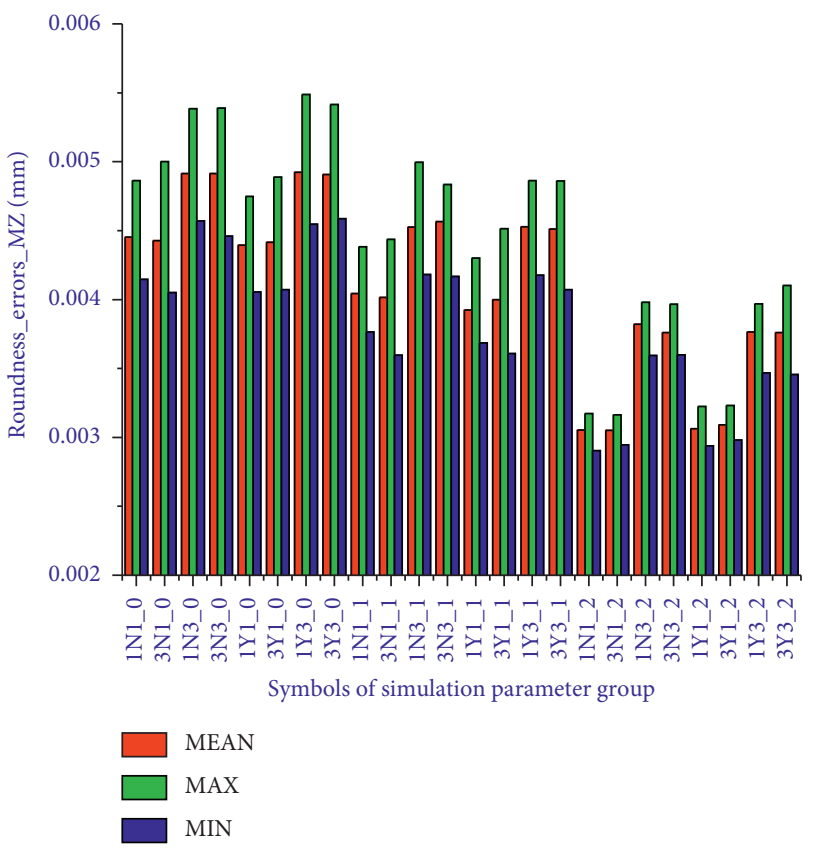

(d)

FIGURE 20: Statistic values of roundness errors of the cylindrical features. (a) Least-square roundness errors. (b) Minimum circumscribed roundness errors. (c) Maximum inscribed roundness errors. (d) Minimum zone roundness errors.

feature, respectively, and $x \times$-LS and $x \times-M Z$ represent the axis straightness errors evaluated by using the least-square criterion [18] and minimum zone criterion [19], respectively. The evaluation results of generatrix straightness errors [20] of generatrix profiles can be seen in Figure 25(b), and the symbol meanings can be seen in Figures 21 and 25(a).

Besides the statistics of the evaluation results of global sizes, calculated sizes, form errors, such as cylindricity errors, roundness errors, generatrix straightness errors, and axis straightness errors, their waviness and roughness evaluation results were also analyzed, as shown in Figure 26.

4.3. Discussion. From Figures 18(a), 18(c), 18(e), and 18(g), the values of MMD are near those of LSD for all extraction strategies, and the influence of waviness and roughness on the MMD and LSD is less, but there are some differences between values of MZC and LSC for each extraction profile. 


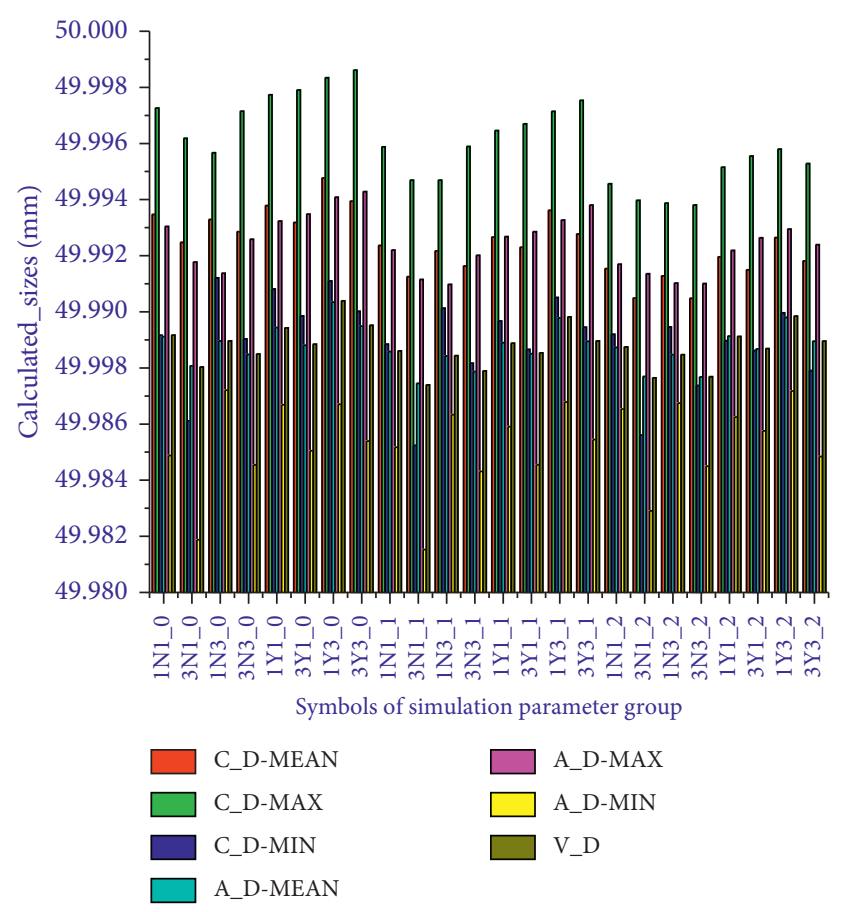

FIGURE 21: Statistic values of calculated sizes of cylindrical features.

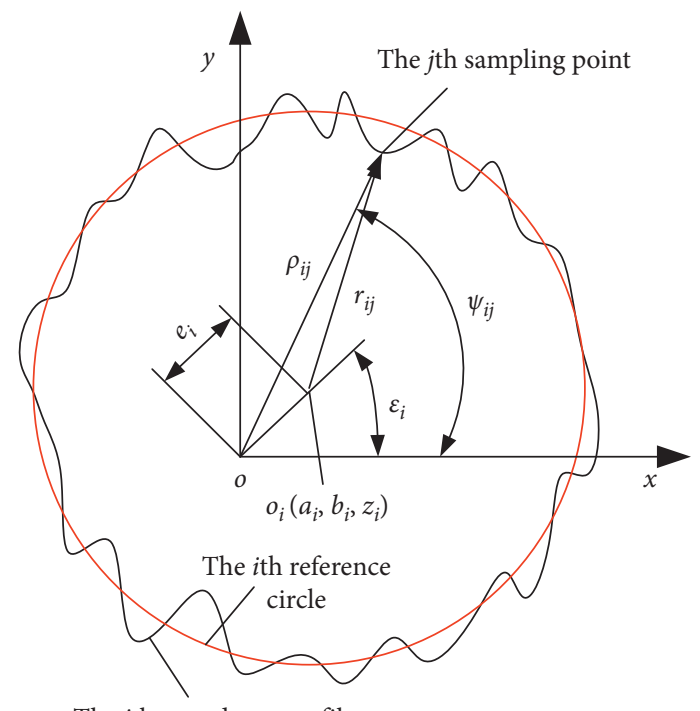

The $i$ th roundness profile

Figure 22: Schematic of center of the reference circle.

From Figure 26, the maximum, minimum, and mean values of waviness and roughness have no much difference for generatrix profiles, but the three values above have much difference for the roundness profile.

For further researching the influence of sampling number on the evaluation results of global sizes and cylindricity errors, we took the sampling number $n_{r}$ or $n_{s}$ in Table 3 as 500, 625, 1000, 1250, 2000, 2500, 5000, and 10000, respectively, which are from the same simulated cylindrical profiles under the sampling number $n_{r}$ or $n_{s}$ being 10000 .

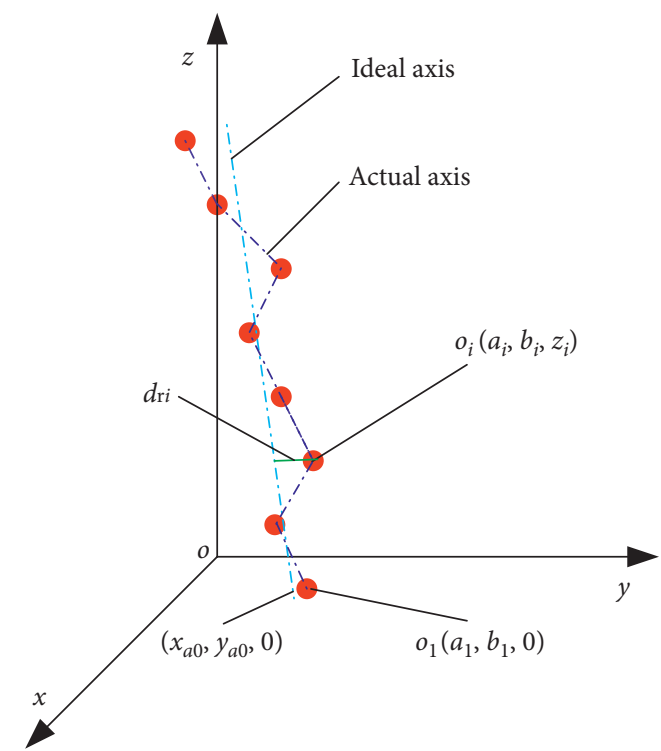

Figure 23: Schematic of axis of the simulated cylinder.

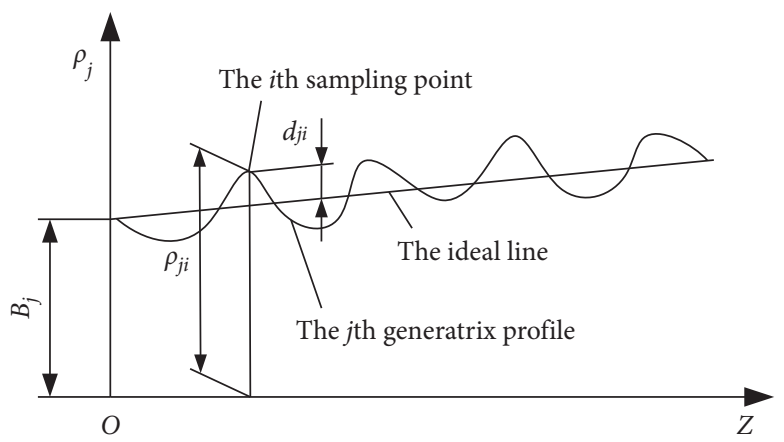

FIgURE 24: Schematic of building the ideal line based on the generatrix profile.

Their corresponding global sizes and cylindricity errors for 8 kinds of simulation parameter symbols and 4 kinds of extraction strategies in Figures 18 and 19 were evaluated. Suppose that the gained global sizes and cylindricity errors are expressed as $D_{\text {ESML }}$ and $C_{\text {ESML }}$, respectively, where subscripts E, S, M, and $\mathrm{L}$ represent extraction strategy of profiles, simulation parameter symbol, evaluation method, and sampling number, respectively. Suppose that E from 1 to 4 means RP, GP, BP, and SP, respectively, S from 1 to 8 corresponds to the symbols 1N1_X, 1N3_X, 3N1_X, 3N3_X, 1Y1_X, 1Y3_X, 3Y1_X, and 3Y3_X, respectively, $M$ from 1 to 4 means LSD (LSC), MCD (MCC), MID (MIC), and MMD (MZC), respectively, and L from 1 to 7 means the sampling points 500, 625, 1000, 1250, 2000, 2500, and 5000, respectively. We can obtain the following $X A_{\mathrm{ML}}$ matrix based on $X R_{\text {SEML }}$ values, where $X R_{\text {SEML }}=D_{\text {SEML }} / D_{\text {SEMI }}$ for global sizes and $C_{\mathrm{SEML}} / C_{\mathrm{SEM} 7}$ for cylindricity errors, $\mathrm{M}=1 \sim 4, \mathrm{~L}=1 \sim 7$ and symbol I means that the sampling number is 10000 : 


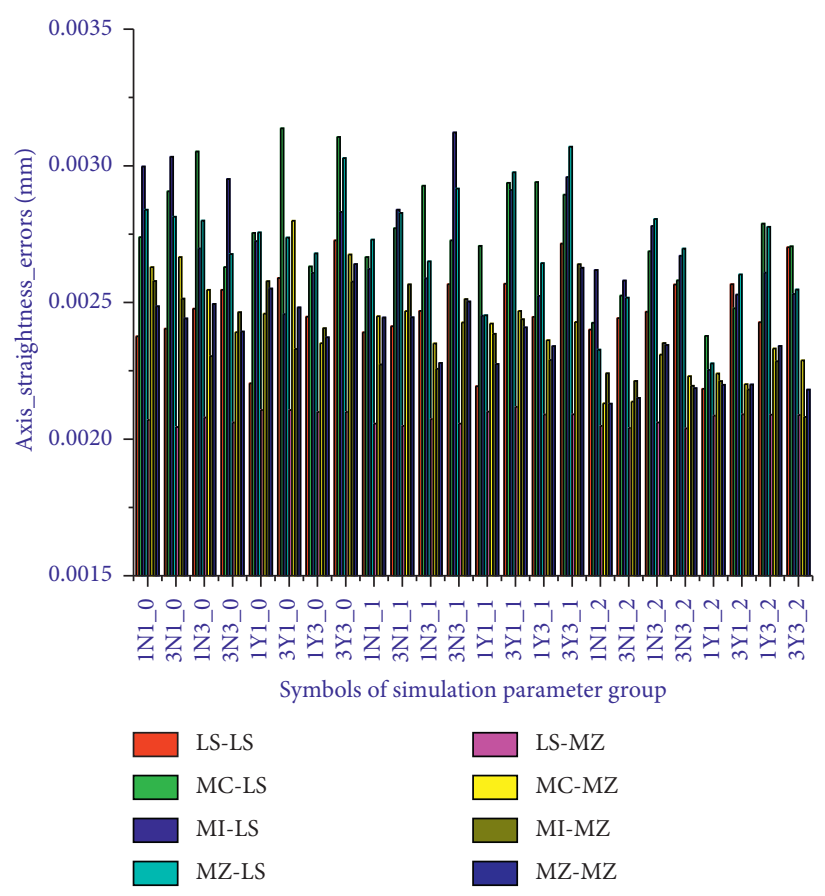

(a)

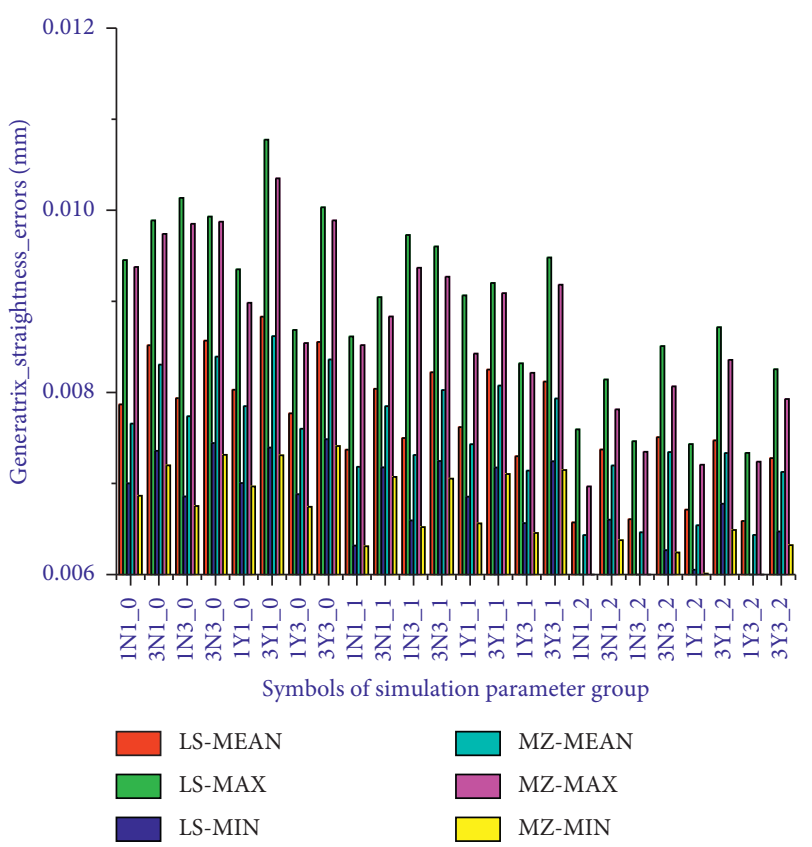

(b)

Figure 25: Statistic values of straightness errors of cylindrical features. (a) Axis straightness errors. (b) Generatrix straightness errors.

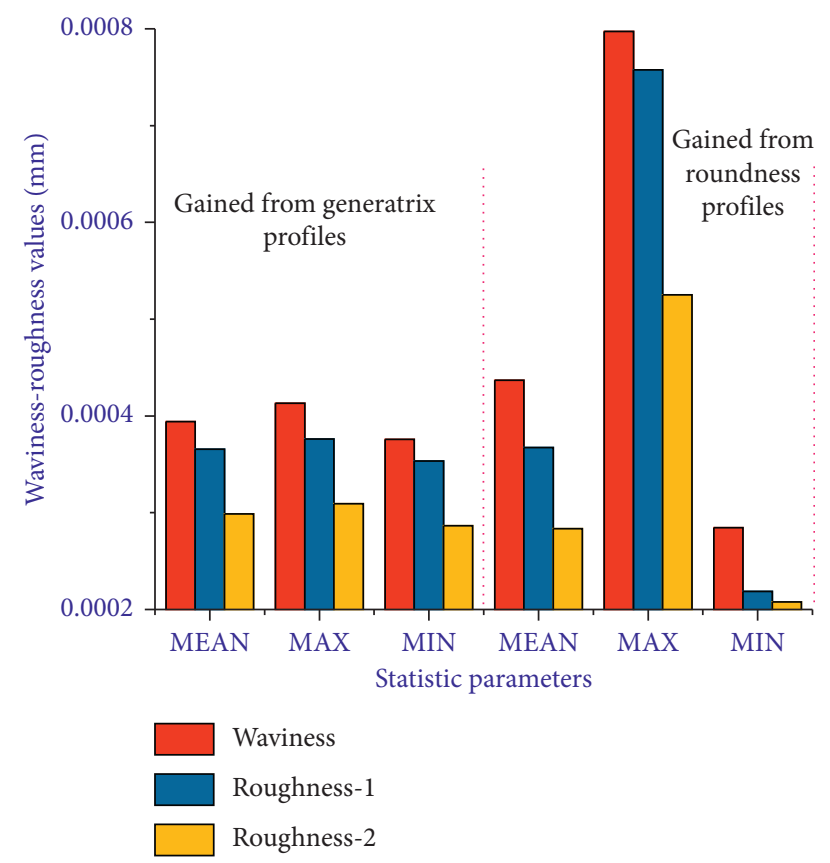

Figure 26: Statistic values of waviness and roughness of cylindrical features. 


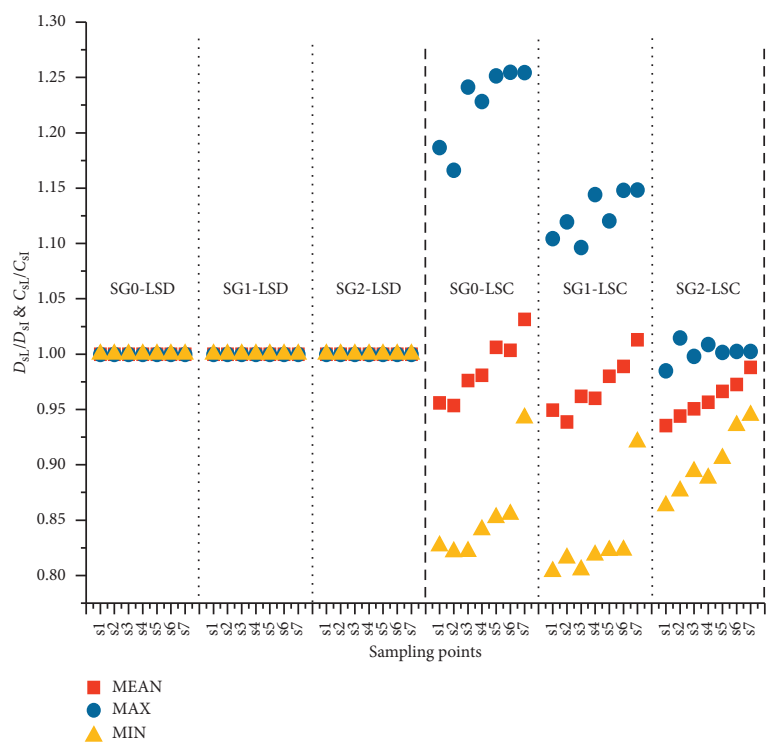

(a)

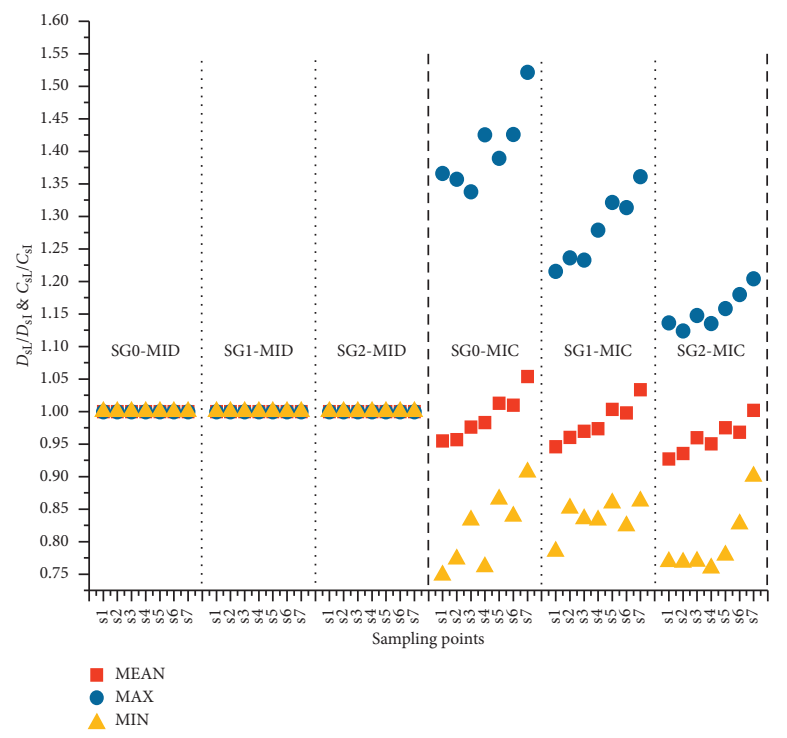

(c)

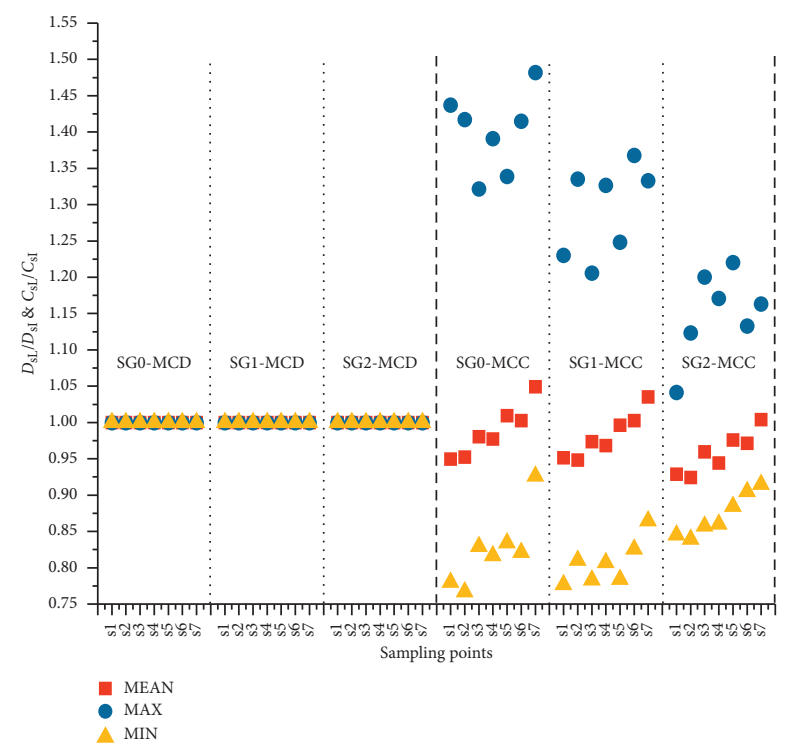

(b)

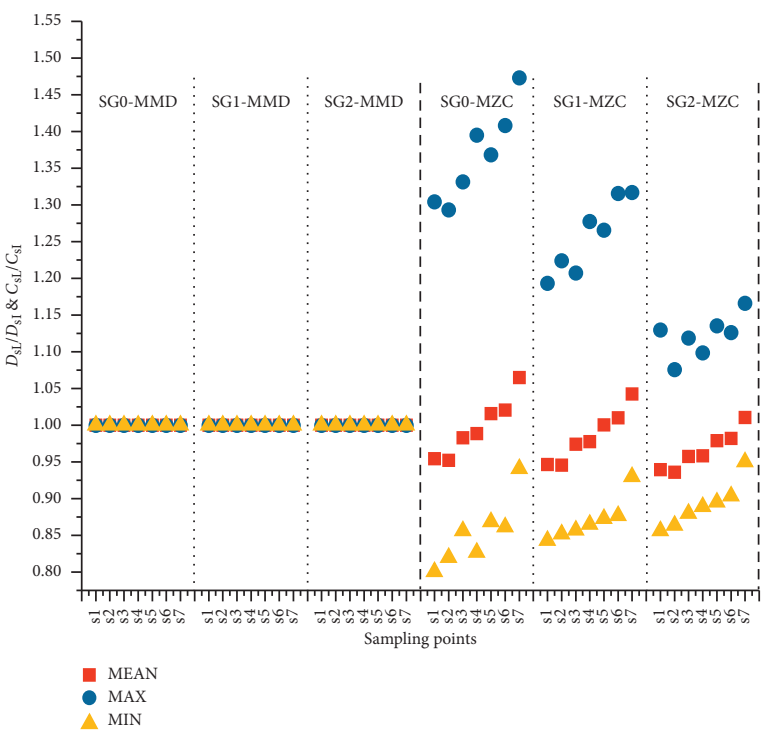

(d)

FIgUre 27: $D_{\mathrm{SL}} / D_{\mathrm{sI}} \& C_{\mathrm{sL}} / C_{\mathrm{sI}}$ of different sampling numbers. (a) LSD and LSC. (b) MCD and MCC. (c) MID and MIC. (d) MMD and MZC.

$$
X A_{\mathrm{ML}}=\left[\begin{array}{llllllll}
X R_{11 \mathrm{ML}} & X R_{12 \mathrm{ML}} & X R_{13 \mathrm{ML}} & X R_{14 \mathrm{ML}} & X R_{15 \mathrm{ML}} & X R_{16 \mathrm{ML}} & X R_{17 \mathrm{ML}} & X R_{18 \mathrm{ML}} \\
X R_{21 \mathrm{ML}} & X R_{22 \mathrm{ML}} & X R_{23 \mathrm{ML}} & X R_{24 \mathrm{ML}} & X R_{25 \mathrm{ML}} & X R_{26 \mathrm{ML}} & X R_{27 \mathrm{ML}} & X R_{28 \mathrm{ML}} \\
X R_{31 \mathrm{ML}} & X R_{32 \mathrm{ML}} & X R_{33 \mathrm{ML}} & X R_{34 \mathrm{ML}} & X R_{35 \mathrm{ML}} & X R_{36 \mathrm{ML}} & X R_{37 \mathrm{ML}} & X R_{38 \mathrm{ML}} \\
X R_{41 \mathrm{ML}} & X R_{42 \mathrm{ML}} & X R_{43 \mathrm{ML}} & X R_{44 \mathrm{ML}} & X R_{45 \mathrm{ML}} & X R_{46 \mathrm{ML}} & X R_{47 \mathrm{ML}} & X R_{48 \mathrm{ML}}
\end{array}\right] .
$$

The mean, maximum, minimum values $D_{\mathrm{sL}} / D_{\mathrm{SI}}$ or $C_{\mathrm{sL}} /$ $C_{\mathrm{sI}}$ and the standard deviation values $D_{\mathrm{sL}} / D_{\mathrm{sI}}-\mathrm{std}$ or $C_{\mathrm{SL}} /$ $C_{\mathrm{SI}^{-}}$-std of $X A_{\mathrm{ML}}$ for each $\mathrm{M}$ and each $\mathrm{L}$ can be gained, as shown in Figures 27-29, and the symbols from s1 to s7 correspond to L from 1 to 7 , respectively; SG means the group of different sampling numbers simulated under 4 kinds of extraction strategies, and the meaning of 0,1 , and 2 after SG can be seen in the horizontal axis in Figure 28.
From Figures 27 and 28, we can see that the sampling points have no much influence on the evaluation results of LSD, MCD, MID, and MMD, and the standard deviation values $D_{\mathrm{SL}} / D_{\mathrm{SI}}{ }^{-s t d}$ of LSD is the least and $D_{\mathrm{SL}} / D_{\mathrm{SI}}$-std of MCD is the largest among 4 kinds of global sizes. From Figures 27 and 29, we also can see that the sampling number has much influence on the evaluation results of LSC, MCC, MIC, and MZC, and the standard deviation 


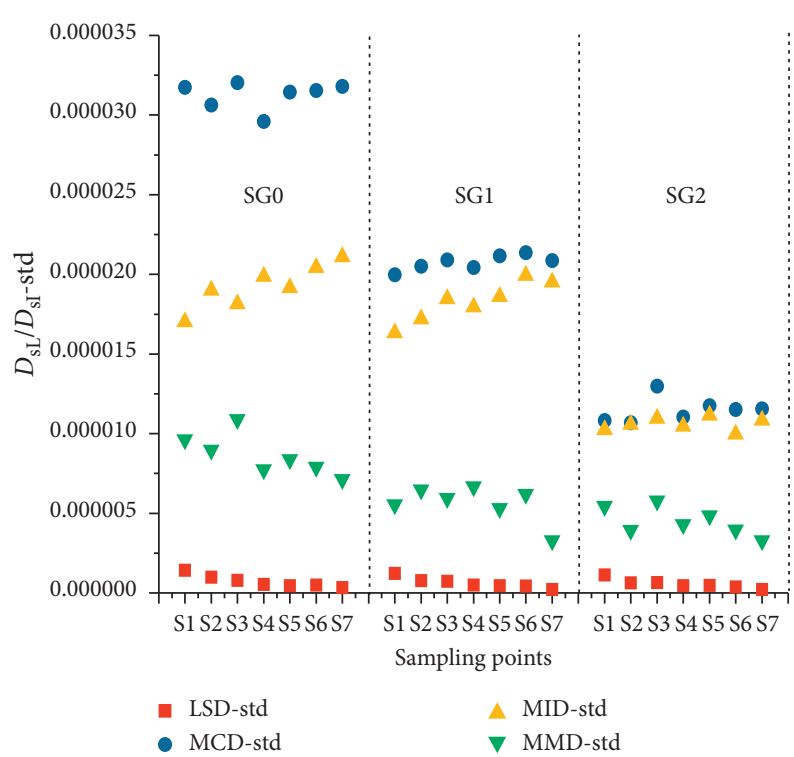

FIGURE 28: $D_{\text {SL }} / D_{\text {SI }}{ }^{- \text {std }}$ of different sampling numbers.

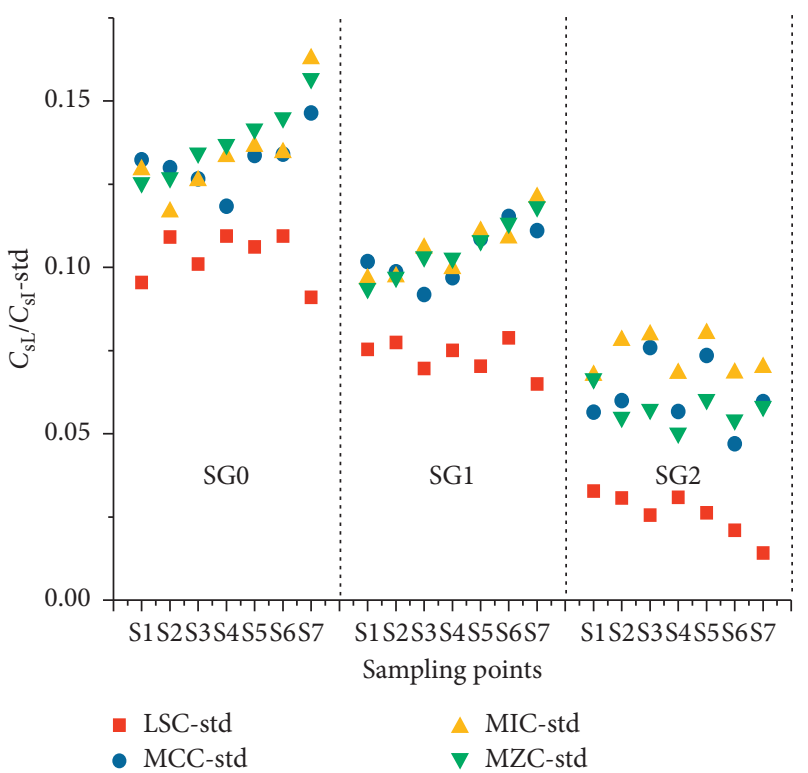

FIGURE 29: $C_{\mathrm{SL}} / C_{\mathrm{SI}} \mathrm{I}^{- \text {std }}$ of different sampling numbers.

values $C_{\mathrm{sL}} / C_{\mathrm{sI}}$-std of LSC is the least. On the other hand, when the extracted profiles for SG2, as shown in Figures 27-29, do not include waviness and roughness profiles, the $D_{\mathrm{sL}} / D_{\mathrm{sI}^{-}}{ }^{-s t d}$ and $C_{\mathrm{sL}} / C_{\mathrm{sI}^{-}}$std of global sizes and cylindricity errors are least among their values of the extracted profiles for SG0, SG1, and SG2, which means that the influence of the sampling number on the measuring results of global sizes and cylindricity sizes is the least when the profiles are extracted under the SG2 condition.

\section{Conclusions}

Based on the analyses of the slideway error, the rotary error of the spindle, and their parallelism error, the simulation models of the slideway profile, the roundness profile, and the parallel profile were built, respectively. The slideway profile consists of the form profile, waviness profile, and roughness profile, and the roundness profile is composed of the eccentric circle, form profile, waviness profile, and roughness profile. According to the built simulation models above and the given values of their corresponding parameters, four kinds of extraction strategies of cylindrical profiles, such as roundness profiles, generatrix profiles, birdcage profile, and single spiral profile, can be simulated, respectively. The determination methods of the modeling parameters of the cylindrical feature were promoted. A cylindrical component with a nominal diameter of $\phi 50 \mathrm{~mm}$, the upper limit deviation of $0 \mathrm{~mm}$, the lower limit deviation of $-0.025 \mathrm{~mm}$, the roundness tolerance of $0.004 \mathrm{~mm}$, the cylindricity tolerance of $0.007 \mathrm{~mm}$, and the roughness arithmetical mean deviation 
$R a$ of $0.4 \mu \mathrm{m}$ was taken as an example for the simulation of its cylindrical feature. Under the given eight sets of the modeling parameter values, eight groups of cylindrical features of the component were obtained, and each group of cylindrical features is characterized by the roundness profiles, generatrix profiles, birdcage profile, and single spiral profile, respectively. Based on the obtained cylindrical features, their global sizes, calculated sizes, cylindricity errors, roundness errors, axis straightness errors, generatrix straightness errors, waviness values, and values of roughness $R a$ were evaluated, and their evaluation values were analyzed through statistics. The simulation and analysis results showed that the modeling parameter values have much influence on the simulation of cylindrical features and their evaluation values of global sizes, cylindricity errors, and roundness errors, and for four extraction strategies of cylindrical profiles, the selected sampling number has no much influence on the global sizes, but has much influence on the cylindricity errors. We can use the built extraction strategy models of cylindrical profiles to further research some issues on the relationship between actual size, form errors, and global sizes.

\section{Data Availability}

The data used to support the findings of this study are available from the corresponding author upon request.

\section{Conflicts of Interest}

The authors declare that they have no conflicts of interest.

\section{Acknowledgments}

This project was supported by the National Natural Science Foundation of China (Grant nos. 51975598 and 51475485).

\section{References}

[1] International Organization for Standardization, "ISO 286-1: 2010," Geometrical Product Specifications (GPS)-ISO Code System for Tolerances on Linear Sizes-Part 1: Basis of Tolerances, Deviations and Fits, https://www.iso.org/standard/ 45975.html? browse =tc, 2010.

[2] International Organization for Standardization, "ISO 1101: 2017," Geometrical Product Specifications(GPS)-Geometrical Tolerancing-Tolerances of Form, Orientation, Location and Run-Out, https://www.iso.org/standard/66777.html? browse $=$ tc, 2017.

[3] International Organization for Standardization, "ISO 1101: 2017," Geometrical Product Specifications (GPS)-Surface Texture: Profile Method-Terms, Definitions and Surface Texture Parameters, https://www.iso.org/standard/10132.html? browse $=\mathrm{tc}, 1997$.

[4] International Organization for Standardization, "ISO 144051:2016," Geometrical Product Specifications (GPS)-Dimensional Tolerancing-Part 1: Linear sizes, https://www.iso.org/ standard/65202.html?browse=tc, 2016.

[5] Z. Zhao, B. Li, G. Zhang et al., "Study on the evaluation of cylinder's global sizes," Precision Engineering, vol. 49, pp. 189-199, 2017.
[6] International Organization for Standardization, "ISO 121802:2011," Geometrical Product Specifications (GPS)-Cylindricity-Part 2: Specification Operators, https://www.iso.org/ standard/53612.html?browse $=$ tc, 2011.

[7] Z. Zhao and Z. Zhao, "Evaluation of cylindrical form errors by mean square approximation method," Acta Metrologica Sinica, vol. 10, pp. 138-143, 1989.

[8] SAC/TC 240, GB/T 1031-2009, Geometrical Product Specifications (GPS)-Surface Texture: Profile method-Surface Roughness Parameters and their Value, http://www.doc88. com/p-3307854669735.html, 2009.

[9] International Organization for Standardization, "ISO 1661021:2011," Geometrical Product Specifications (GPS)-FiltrationPart 21: Linear Profile Filters: Gaussian Filters, https://www. iso.org/standard/50176.html?browse=tc, 2011.

[10] L. Lévesque, "Nyquist sampling theorem: understanding the illusion of a spinning wheel captured with a video camera," Physics Education, vol. 49, no. 6, pp. 697-705, 2014.

[11] K. Lee, S. Cho, and S. Asfour, "Web-based algorithm for cylindricity evaluation using support vector machine learning," Computers \& Industrial Engineering, vol. 60, no. 2, pp. 228-235, 2011.

[12] X. Lei, H. Song, Y. Xue, J. Li, J. Zhou, and M. Duan, "Method for cylindricity error evaluation using geometry optimization searching algorithm," Measurement, vol. 44, no. 9, pp. 1556-1563, 2011.

[13] W. Ni and Z. Yao, "Cylindricity modeling and tolerance analysis for cylindrical components," The International Journal of Advanced Manufacturing Technology, vol. 64, pp. 867-874, 2013.

[14] M. Dovica and A. Végh, "Comparison of the cylindricity deviation using different evaluation methods," American Journal of Mechanical Engineering, vol. 1, no. 7, pp. 339-342, 2013.

[15] W. Sui and D. Zhang, "Four methods for roundness evaluation," Physics Procedia, vol. 24, pp. 2159-2164, 2012.

[16] A. J. Mohammad, L. Gopinath, and S. Ravishankar, "Mathematical approaches in evaluation of circularity measurement," International Journal of Engineering Research \& Technology, vol. 5, pp. 572-576, 2016.

[17] Z. Zhao, J. Xi, X. Zhao, G. Zhang, and M. Shang, "Evaluation of the calculated sizes based on the neural network regression," Mathematical Problems in Engineering, vol. 2018, Article ID 4078456, 11 pages, 2018.

[18] J. Luo and Q. Wang, "A method for axis straightness error evaluation based on improved artificial bee colony algorithm," The International Journal of Advanced Manufacturing Technology, vol. 71, no. 5-8, pp. 1501-1509, 2014.

[19] Q. Zhang, K. C. Fan, and Z. Li, "Evaluation method for spatial straightness errors based on minimum zone condition," Precision Engineering, vol. 23, no. 4, pp. 264-272, 1999.

[20] C. Cui, T. Li, L. A. Blunt et al., "The assessment of straightness and flatness errors using particle swarm optimization," Procedia CIRP, vol. 10, pp. 271-275, 2013. 